\title{
Representative elementary volumes, hysteresis and heterogeneity in multiphase flow from the pore to continuum scale
}

\author{
S. J. Jackson ${ }^{1,2}$, Q. Lin ${ }^{2}$ and S. Krevor ${ }^{2}$ \\ ${ }^{1}$ CSIRO Energy, Private Bag 10, Clayton South, Victoria 3169, Australia \\ ${ }^{2}$ Department of Earth Science \& Engineering, Imperial College London, London, U.K.
}

\section{Key Points:}

- $\mu \mathrm{m}$ - cm scale observations during steady-state drainage \& imbibition in Bentheimer sandstones used to validate continuum modelling concepts.

- Porosity \& capillary pressure REV $<2 \mathrm{~mm}^{3}$. Saturation REV is fractional flow dependent from $3.4-157.5 \mathrm{~mm}^{3}$. REV uncertainty key in model evaluation.

- 3D models with REV heterogeneities \& hysteresis predict fluid configuration, connectivity \& relative permeability variations between samples.

Corresponding author: S.J. Jackson, samuel.jackson@csiro.au 


\begin{abstract}
Representative elementary volumes (REVs) and heterogeneity are key concepts in continuum multiphase flow, yet their manifestation from the pore-scale and associated impacts with the flow regime are not well understood. We use a multi-scale experimental and modelling approach to elucidate the role of REVs, hysteresis and heterogeneity in multiphase flow in two distinct water-wetting Bentheimer sandstones. Experimental observations during steady-state drainage \& imbibition resolve the pore space at $6 \mu \mathrm{m}$ across a field of view up to $12 \times 12 \times 65 \mathrm{~mm}$ with simultaneous measurements of differential pressure during fluid flow. The REV for porosity and capillary pressure is $\approx 2 \mathrm{~mm}^{3}$ for both samples, with $5 \%$ relative uncertainty. In contrast, due to macroscopic capillary pressure heterogeneities, the REV for saturation varies between $3.4-157.5 \mathrm{~mm}^{3}$, dependent on the fractional flow. Accurate knowledge of this REV uncertainty is critical in assessing continuum scale model validity and predictiveness. We validate the Land trapping model directly by predicting observed non-wetting fluid connectivity at multiple imbibition states to within the REV uncertainty for homogeneous media. With this, observed hysteresis in the measured relative permeability is entirely removed when considering the connected saturation, validating conceptual hysteresis models based on connected pathway flow. REV scale heterogeneities in capillary pressure are shown to impact flow and trapping characteristics; when built into 3D continuum scale numerical models with hysteresis we are able to predict the variations in trapping and relative permeability observed between the two samples with different heterogeneity structures. The experimental dataset herein provides an excellent benchmark for future development of continuum scale modelling frameworks.
\end{abstract}

\title{
1 Introduction
}

In order to tractably model large scale subsurface fluid flow, the porous rock-fluid system is treated as a continuum (Bear, 1989). The prevailing continuum framework makes use of Darcy's law, and defines macroscopic properties - porosity, permeability, saturation, capillary pressure, relative permeability and trapping, which defines hysteresis in the properties. These properties represent the aggregate behaviour of fluids moving through a capillary network formed by the pores and fractures of the rock strata. The approach underpins commercial oil and gas production (Walsh \& Lake, 2003), groundwater management (Todd, 2005), the cleanup of subsurface contaminants radioactive waste and NAPL (Schwartz, 2009; Finsterle et al., 2014), and the large scale mitigation of climate change through subsurface $\mathrm{CO}_{2}$ storage (S. Krevor et al., 2019).

With respect to two phase fluid flow, there remain a number of outstanding questions about the relationship between the fluid dynamics within the pores of the rocks and their upscaled, continuum, manifestation. The continuum approach relies on the concept of a representative elementary volume, or REV. While the REV for porosity and permeability has been exhaustively studied (see for example Arns et al. (2005); Hilfer and Lemmer (2015); Zhang et al. (2000); Al-Raoush and Papadopoulos (2010)), the REV for multiphase flow properties, relative permeability and capillary pressure are not understood (R. Armstrong et al., 2014). Similarly, pore-scale fluid morphology has long been invoked as the underlying cause for hysteresis in continuum properties of relative permeability and capillary pressure (Haines, 1930; Land, 1968; Hassanizadeh \& Gray, 1980; Reeves \& Celia, 1996; McClure et al., 2016). One of the earliest and most widely used trapping models, the Land model, correlates hysteresis with the fraction of fluid phase that is connected vs. isolated as residually trapped ganglia within the pores (Land, 1968). While the model fits well to observations in cm-scale rock cores, the validity of the conceptual picture at the pore-scale is undetermined. Finally, the continuum properties of rock systems are typically measured on rock cores of length scale $0.1 \mathrm{~m}-1 \mathrm{~m}$. However, heterogeneity in continuum properties at scales even smaller than the rock cores can, and 
perhaps usually do, place leading order controls on flow with field scale manifestations (Shook et al., 1992; Ringrose et al., 1993; Ringrose \& Corbett, 1994; Zhou et al., 1981; Reynolds \& Krevor, 2015; Reynolds et al., 2018; Jackson et al., 2018). There is no consensus on the extent to which heterogeneity in multiphase flow properties at these small scales must be accounted for in upscaled models.

The major obstacle to answering these questions has been in the difficulty in observing small scale fluid displacement and distribution in rocks simultaneous with the measurement of continuum scale properties. Even with the recent availability of laboratory and synchrotron X-ray CT direct links between pore-scale fluid properties and their continuum manifestations are challenging (Blunt et al., 2013). They require a combination of imagery at a spatial resolution of micrometres simultaneous with measurements across samples of $\mathrm{cm}$-scale dimensions. There are direct trade-offs inherent in the physics of X-ray CT between field of view and spatial resolution. As a result, few studies have measured pore-scale properties simultaneous with, e.g., relative permeability. Notably (Gao et al., 2017; Lin et al., 2018, 2019) have measured relative permeability and capillary pressure simultaneous with a resolution of the pore-scale fluid distribution, demonstrating the potential of this approach.

In this work, we address the challenges in linking pore-scale fluid dynamics with their continuum manifestations with a multi-scale experimental and modelling approach. We perform steady-state drainage and imbibition core flooding experiments in two distinct, cm-scale Bentheimer cores with detailed X-Ray CT imaging and simultaneous differential pressure measurements. The sample size is optimised to allow micron resolution CT imaging across hundreds of REVs, resolving the interfaces between fluid phases and their connectivity, whilst allowing the determination of continuum scale multiphase flow properties - relative permeability, capillary pressure and trapping. This data is used to develop and validate hysteretic, continuum scale models, evaluating several base assumptions (1) the representative elementary volume for single and multiphase flow properties, (2) the relationship between hysteresis and connected fluid phase saturations (3) the link between heterogeneity in capillary pressure characteristics and the impact on average flow, and flow properties, across the scales at which measurements would typically be obtained. In this way, the continuum scale models are directly validated and compared with pore- and continuum information in a multi-scale analysis.

\section{Experimental methods}

\subsection{Core flooding}

We perform steady-state core flooding experiments with micro-CT imaging on two Bentheimer sandstone cores of diameter $12.35 \mathrm{~mm}$ (cored with a $12.7 \mathrm{~mm}, 1 / 2$ " drill bit), and length $73.2 \mathrm{~mm}$ and $64.7 \mathrm{~mm}$, with core-averaged porosities of 0.203 and 0.223 respectively. The shallow-marine Bentheimer sandstone consists of around 95\% quartz with minor feldspar and clay and has a well-sorted grain size distribution (Peksa et al., 2015).

Typical core samples used for multiphase flow experiments are shown in Figure 1a, with the core used in this experimental campaign shown in the centre. Small millimetresized cores are typically used for pore-scale flow experiments, since they allow detailed micro-CT imaging at resolutions of 1-4 microns (Andrew et al., 2014b; Singh et al., 2016), imaging volumes typically around $1-100 \mathrm{~mm}^{3}$. For Bentheimer sandstones, the representative elementary volumes (REV) for porosity is typically between 1 and $60 \mathrm{~mm}^{3}$ (Halisch, 2013; Herring et al., 2013), but can be larger for multiphase properties such as saturation (Herring et al., 2013). This means that for many properties an REV is not reached on samples often used in experiments with micro CT imaging.

At larger scales (Figure 1a top), samples used in special core analysis allow the derivation of continuum flow properties (McPhee et al., 2015). With the inclusion of REV scale 


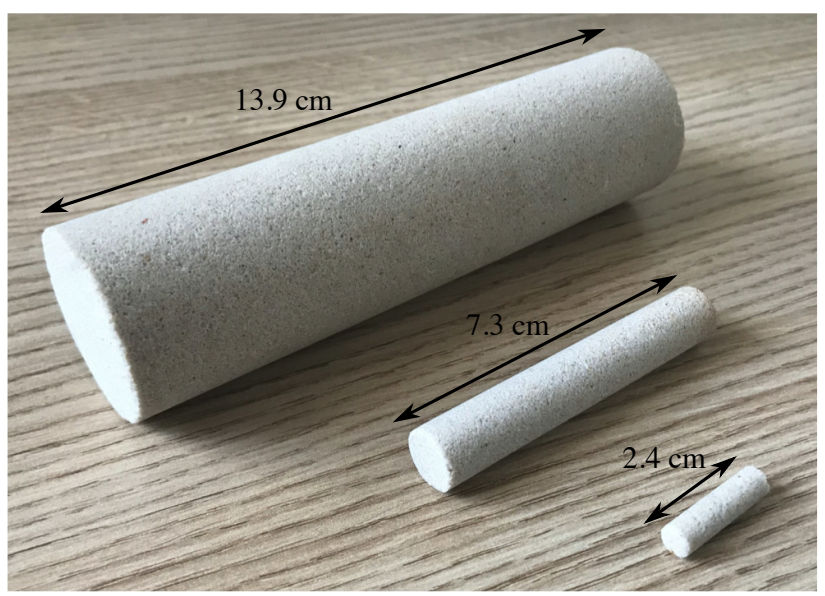

(a)

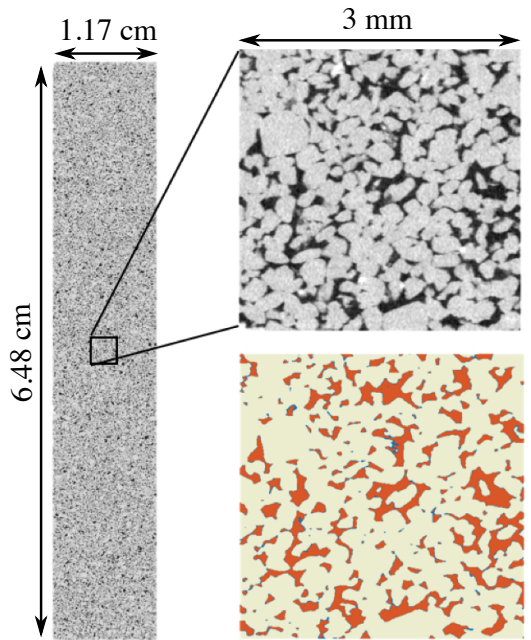

(b)

Figure 1. (a) Typical X-Ray CT-Core flooding samples sizes (Bentheimer sandstone cores). The smallest cores typically have diameters of $4-6 \mathrm{~mm}$. The largest cores typically have diameters of $35-65 \mathrm{~mm}$. The sample used in this work (middle) has dimensions in between that allow pore-scale imaging (i.e. $O(\mu \mathrm{m})$ resolution) across continuum scales (i.e. several REVs). (b) Raw, unfiltered Micro-CT image of the air-saturated middle sample from (a), showing raw and segmented zoomed in regions on the top and bottom respectively.

structural heterogeneity, intrinsic multiphase flow properties can be obtained even in highly heterogeneous samples (Jackson et al., 2018). However, in these approaches, pore-scale displacement mechanisms cannot be directly visualised, which hinders continuum scale model development. For example, unvalidated assumptions often have to be made on the multiphase flow regime from which to construct continuum models, i.e. connected pathway flow and capillary equilibrium.

In this work, the sample size is optimised to allow detailed micron resolution CT imaging across hundreds of REVs. The Bentheimer sandstone samples in this study have permeabilities of $1.636 \mathrm{D} \pm 0.025 \mathrm{D}$ and $0.681 \mathrm{D} \pm 0.006 \mathrm{D}$ respectively, average pore diameters of $\approx 80 \mu \mathrm{m}$ (Peksa et al., 2015) and total volumes of $8769 \mathrm{~mm}^{3}$ and $7750 \mathrm{~mm}^{3}$ respectively. Using a full core scan resolution of $6 \mu \mathrm{m}$ means that the fluid phases in individual pores can be resolved, whilst allowing 100s of REVs to be imaged. Imaging methods, analysis and modelling from both pore and continuum scales can then be applied simultaneously on the same core, allowing the inclusion of complementary information.

In the core flooding experiments, brine (3.5wt.\% KI) and decane (99.9\% pure, SigmaAldrich) are used as wetting and non-wetting phases respectively, with an interfacial tension of $\approx 51.2 \mathrm{mN} / \mathrm{m}$ (for the pure DI water - decane system (Georgiadis et al., 2011), with maximum $\pm 0.5 \mathrm{mN} / \mathrm{m}$ for the addition of small KI salt concentrations to the water (Aveyard \& Saleem, 1976)). The experimental pore pressure is maintained at 1.5MPa, and the temperature $\mathrm{T}$ at $30{ }^{\circ} \mathrm{C}$ (core 1 experiment, $\mathrm{T}=30.11{ }^{\circ} \mathrm{C} \pm 0.46{ }^{\circ} \mathrm{C}$, core 2 experiment, $\mathrm{T}=29.90^{\circ} \mathrm{C} \pm 0.44^{\circ} \mathrm{C}$ ). These conditions result in estimated fluid viscosities for brine and decane as $7.83 \times 10^{-4}$ Pa.s (Goldsack \& Franchetto, 1977) and 8.03 $\times 10^{-4}$ Pa.s (Linstrom, 1997) respectively, and densities of $1023.2 \mathrm{~kg} \cdot \mathrm{m}^{-3}$ (Ghafri et al., 2012) and $723.8 \mathrm{~kg} \cdot \mathrm{m}^{-3}$ (Linstrom, 1997) respectively. These fluids are chosen for their relative stability in the core-flooding experiments (as opposed to gaseous flows) and their analogy to other immiscible fluid pairs found in subsurface flows, for example in carbon 
sequestration (supercritical $\mathrm{CO}_{2}$-brine), oil recovery (Crude oil - brine) and contaminant transport (NaPLs - brine).

In order to assess the impact of rock heterogeneities at the pore and continuum scales, the cores are flooded at low capillary number (total flow rate, $Q_{t o t}=0.1 \mathrm{ml} \cdot \mathrm{min}^{-1}$ ), at near unity viscosity ratio $\left(\mu_{n w} / \mu_{w}=1.025\right)$. The pore-scale capillary number at the final fractional flow of oil during drainage $f_{n w}=1$ is $N_{c p}=\frac{u \mu}{\sigma}=2.2 \times 10^{-7}$, where $u$ is the Darcy velocity, $\mu$ is the invading phase dynamic viscosity and $\sigma$ is the fluid-fluid interfacial tension. A macroscopic, continuum scale capillary at the same fractional flow is $N_{c m}=\frac{\Delta P}{L} \frac{H}{\Delta P_{c}} \approx 0.25$, where $\Delta P$ is the core-averaged pressure drop over length $L$ and $\Delta P_{c}$ is the variation in characteristic capillary pressure over heterogeneity length scale $H$ (Virnovsky et al., 2004). Here, $\Delta P_{c}$ is taken as $1.5 \mathrm{kPa}$ (Jackson et al., 2018), over a length scale $H$ equal to the core radius. The macroscopic capillary number indicates the flow is nearing the capillary limit, far below the transition to viscous-dominated flow reported typically in the range $N_{c m}=10-100$ (Virnovsky et al., 2004; Jackson et al., 2018). Macroscopic structural heterogeneities will therefore dominate the flow regime, controlling the flow path and potential (Pini \& Benson, 2013). At the pore-scale, the flow is at capillary equilibrium; with the near unity viscosity ratio and low $N_{c p}$ it should exhibit connected pathway flow at steady-state. Intermittent fluid connectivity generally occurs in non-negligible volume fractions only when $\mu_{n w} / \mu_{w}<<1$, i.e. in gas-water systems (Berg et al., 2016; Kogure et al., 2017; Reynolds et al., 2017; Gao et al., 2017; Spurin et al., 2019a, 2019b). We note there may be some small-scale intermittent fluctuations in the fluid distribution, highlighted by the pressure signals in the SI, that are not captured by the time-averaged nature of the scanning.

The core flooding experimental procedure is detailed in the supporting information, and summarised here. We use a similar approach to that described in (Lin et al., 2018), optimised for a large sample size and core stability during long scans. The core is placed in the core-holder setup shown in SI Figure 1 with a confining pressure of $4.5 \mathrm{MPa}$ to prevent fluid-bypass between the core and viton sleeve. A full-core scan of the air-saturated core is first taken, using the imaging methodology described in section 2.2. The core is then saturated with high-salinity brine (30wt.\% KI) by flowing at least 100 pore volumes through at high-flow rate $\left(Q_{t o t}=5 \mathrm{ml} . \mathrm{min}^{-1}\right)$ with a back-pressure (pore-pressure) of $1.5 \mathrm{MPa}$, to fully displace any air in the system. A full-core scan is then taken for the differential imaging procedure. The working fluid brine $(3.5 \mathrm{wt} . \% \mathrm{KI})$ is then pumped through the core, fully displacing the high-salinity brine, and the absolute permeability is measured. Following this, decane and brine are co-injected into the core at constant total flow rate varying the fractional flow in a drainage and imbibition process. At each fractional flow, once the differential pressure has stabilised across the core (i.e. once $\Delta P / \Delta t<$ $0.03 \mathrm{kPa} \cdot \mathrm{h}^{-1}$ measured over a 6 hour period, with at least 10 hours of flow initially. Note the transducer accuracy is $\pm 0.03 \mathrm{kPa}$ ) a full-core scan is taken followed by zoom-in, region of interest scans. Graphs of the differential pressure through time for both cores are shown in the supporting information Figure 8.

\subsection{X-Ray micro-CT imaging and processing}

Once steady-state has been reached at each fractional flow a full core X-Ray microCT scan is taken using a flat panel detector in a Zeiss Versa 510 X-Ray microscope at a resolution of $6 \mu \mathrm{m}$. Full details of the scanning and image processing along with rationale can be found in the supporting information. In summary, 11 and 10 individual scans are taken at increasing vertical locations in each core, to cover a total volume after processing of $1950 \times 1950 \times 10800$ voxels $(11.7 \mathrm{~mm} \times 11.7 \mathrm{~mm} \times 64.8 \mathrm{~mm})$ and $1900 \times 1900$ $\times 9550$ voxels $(11.4 \mathrm{~mm} \times 11.4 \mathrm{~mm} \times 57.3 \mathrm{~mm})$ in core 1 and 2 , respectively. Approximately $4 \mathrm{~mm}$ is lost from the images near the inlet and outlet of the core due to noise from the end-pieces and the cone angle of the X-Ray beam. There is significant overlap between each scan to allow registration and merging of the full image. Each individual 
scan image is normalised to pre-selected references to obtain consistent grey-scale values throughout the core, after which two weak non-local means filters are applied to the image for noise reduction (Buades et al., 2007). Scanning of the full core takes approximately 11 hours.

After each full-core scan, a selection of zoom-in, region of interest scans are performed to facilitate the interfacial curvature measurements and model validation (discussed in the SI). A $4 \mathrm{x}$ microscope objective lens is used to aquire each image, with the detector position varied to achieve a $3.5 \mu \mathrm{m}$ resolution and $2 \mu \mathrm{m}$ resolution image, each containing $1000{ }^{3}$ voxels. These scans take $18 \mathrm{hrs}$ and $24 \mathrm{hrs}$, respectively. After image acquisition and post-processing (normalisation, registration, merging and filtering), the images are segmented. The full core air saturated and high-salinity brine images are used to accurately segment the pore space into micro and macro porous regions using the differential imaging methodology of (Lin et al., 2016, 2017; Gao et al., 2017). A key aspect of this method is that the macro porous regions are not over-segmented to match external measures when image resolution is limited, as is common when segmenting macro porosity based solely from an air-saturated image of the rock core.

To segment the fluid phases during the drainage and imbibition fractional flows, a marker based watershed algorithm is used (Andrew et al., 2014a; Jones et al., 2007). Using watershed segmentation, fluid-fluid interfaces can be accurately defined and partialvolume layers (i.e. unphysical water layers) and avoided. The segmented pore-space images can be used directly to generate continuum scale, macroscopic quantities, such as averaged porosities and saturations:

$$
\bar{\phi}=1-\frac{\sum_{i}^{N_{v}} \delta_{k, 1} V_{i}}{\sum_{i}^{N_{v}} V_{i}}, \quad \bar{S}_{j}=\frac{\sum_{i}^{N_{v}} \delta_{k, j+1} V_{i}}{\sum_{i}^{N_{v}} V_{i} \bar{\phi}}
$$

where $\bar{\phi}$ is the average porosity, $\bar{S}_{j}$ is the average phase saturation ( $j=1,2$ for non-wetting and wetting phase respectively), $N_{v}$ is the total number of voxels in the averaging volume, $\delta_{k, j}$ is the Kronecker delta function and $V_{i}$ is the volume of each voxel. For the delta function, $k$ is the specific voxel label, with 1, 2, 3 referring to grain, non-wetting phase and wetting phase labels, respectively. Note, specific micro and macro porosities (along with connected/disconnected fluid phases, see below) can be derived in a similar manner using an appropriately labelled image

Following segmentation, the non-wetting phase connectivity is computed from the inlet to the outlet across the entire image using the "Axis connectivity" function in Avizo 9.5. Any voxels with a common vertex are considered connected. Segmentation and subsequent connectivity uncertainty is discussed further in section 4.2 and in detail in the SI.

To highlight the nature of the Bentheimer samples used in this study, and the robustness of the image processing work-flow, the imaged-based porosity for both cores is displayed in Figure 2. Alongside this, an independent measurement of porosity obtained using X-ray imaging from a conventional medical CT scanner with difference imaging is also shown for validation purposes. The porosity was obtained using differential images of the air and brine saturated core at a resolution of $150 \mu \mathrm{m} \times 150 \mu \mathrm{m} \times 500 \mu \mathrm{m}$, which is volumetrically averaged to the slice-averaged values shown in Figure 2. The porosity is calculated through $\phi_{m C T}=\left(C T_{\text {brine }}-C T_{a i r}\right) /\left(C T_{\text {brine, ref }}-C T_{a i r, r e f}\right)$, where the references values of brine and air in the core-holder assembly are $991.9 \mathrm{HU}$ and $-994.4 \mathrm{HU}$ respectively. The medical CT total porosity and segmented micro CT porosity agree well with slice averaged $L_{1}$ error norms of $0.83 \%$ and $1.03 \%$ for core 1 and 2 respectively, highlighting the effectiveness of the image normalisation, merging and differential imaging segmentation method. Further details of the method can be found in the supporting information. 


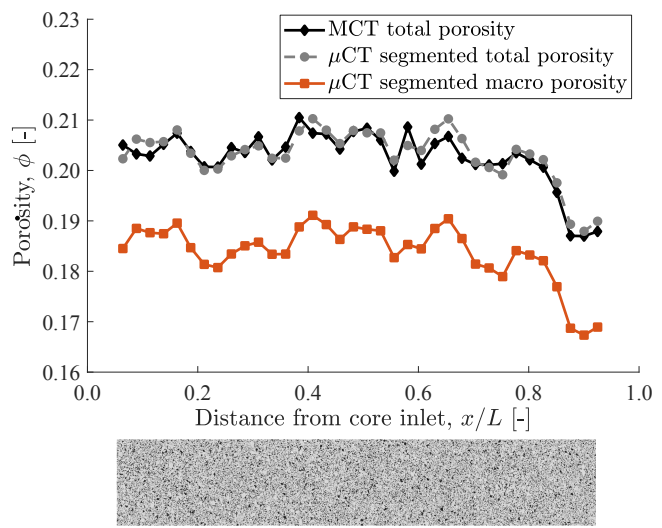

(a)

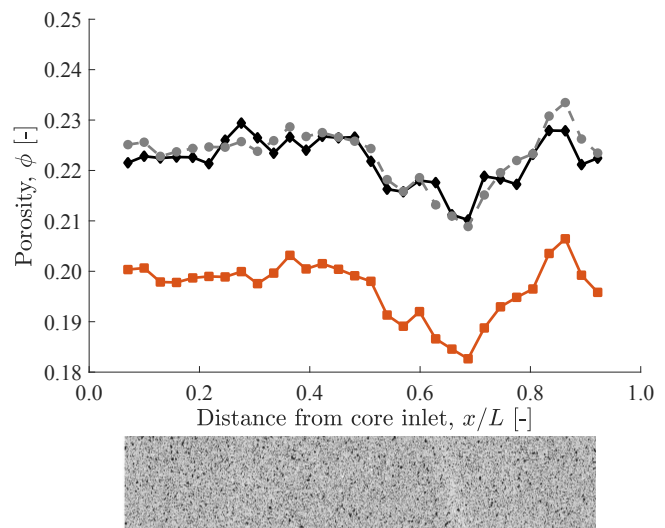

(b)

Figure 2. Slice averaged porosities for the two Bentheimer samples calculated using segmented $\mu \mathrm{CT}$ images and differential medical CT images. (a) Core 1 , slice width $=1.8 \mathrm{~mm}$. (b) Core 2 , slice width $=1.9 \mathrm{~mm}$. Underneath the porosity graphs is the central slice of the raw, unfiltered air-saturated scan for each core, highlighting heterogeneous regions.

The slice average porosities in Figure 2 highlight the heterogeneity in both cores. In core 1, there is a gradual tailing in the porosity towards the outlet of the sample, seen in both medical and micro-CT measured porosities. In core 2 , there is a defined layer around $2 / 3$ rds of the way through the core. This layer is off-perpendicular to the flow direction (left to right in the image), and represents a significant barrier to flow, discussed later in section 4.2. The variation in total porosity through the samples is maintained almost entirely by the macro porous regions, with the micro porous regions having a near constant porosity through the core.

\section{Theory and modelling methods}

In the following sections we describe the continuum scale theory and modelling methods that allow analysis and prediction of the experimental observations. An expanded discussion, further equations and numerical verification can be found in the SI.

\subsection{The continuum framework}

In the continuum modelling framework of multiphase flow we utilise the multiphase extension to Darcy's law (Muskat \& Meres, 1936) with the conservation of mass:

$$
\boldsymbol{u}_{\alpha}=\frac{-\boldsymbol{K} k_{r \alpha}\left(S_{\alpha}\right)}{\mu_{\alpha}} \nabla \psi_{\alpha}, \quad \phi \frac{\partial S_{\alpha}}{\partial t}+\nabla \cdot \boldsymbol{u}_{\alpha}=Q_{\alpha}
$$

Here, $\alpha$ refers to the phase (non-wetting or wetting), $\boldsymbol{u}_{\alpha}$ is the Darcy velocity, $\boldsymbol{K}$ is the absolute permeability tensor, $k_{r \alpha}$ is the relative permeability, $\mu_{\alpha}$ is the viscosity, $S_{\alpha}$ is the fluid saturation, $P_{\alpha}$ is the phase pressure, $\psi_{\alpha}=P_{\alpha}-\rho_{\alpha} g z$ is the flow potential, $g$ is the component of gravitational acceleration in the vertical $z$ direction, $\phi$ is the porosity, $t$ is time and $Q_{\alpha}$ is an external source or sink of phase $\alpha$ (Niessner et al., 2011). To close the system of equations the the following equations are used:

$$
S_{w}+S_{n}=1, \quad P_{n}-P_{w}=P_{c}=P_{c}\left(S_{w}\right), \quad k_{r \alpha}=k_{r \alpha}\left(S_{\alpha}\right)
$$


where $n$ refers to non-wetting phase, $w$ to the wetting phase, and $P_{c}$ is the macroscopic capillary pressure. The macroscopic multiphase extension of Darcy's law (2) can be derived by directly homogeneizing Stokes flow at the pore-scale over a suitably defined representative elementary volume (Hassanizadeh \& Gray, 1980; Whitaker, 1986; Bourgeat, 1997) under the following assumptions: inertial and (macroscopic) viscous effects are negligible (including viscous coupling between the phases), the solid phase is composed of incompressible grains, and the average fluid saturation evolves slowly in space and time through the REV (Blunt, 2017). Similar concepts can be used to derive the macroscopic equation of mass conservation, energy conservation if heat transfer is to be considered and solid-phase momentum conservation if considering porous media deformation (Hassanizadeh \& Gray, 1980).

The solution of the mass and momentum equations 2 requires constitutive functional relationships between the relative permeability $k_{r \alpha}$, capillary pressure $P_{c}$ and saturation $S_{\alpha}$, which are usually derived empirically from experiments. These functions are known to exhibit hysteresis (i.e. different functional forms during drainage and subsequent imbibition) (Oak, 1980) due to their non-unique relationship with total saturation $\left(S_{\alpha}\right)$. They can also exhibit flow rate dependence due to the impact of heterogeneities (Jackson et al., 2018). The drainage relative permeability for the non-wetting $k_{r n w}$ and wetting phase $k_{r, w}$ are parametrised here using power-law functions:

$$
k_{r n w}=k_{r n w}\left(S_{w i r r}\right)\left(1-S_{w}^{*}\right)^{n}, \quad k_{r w}=\left(S_{w}^{*}\right)^{m}
$$

where $S_{w}^{*}$ is the water saturation normalised to the irreducible $S_{w i r r}$, i.e. $S_{w}^{*}=\left(S_{w}-\right.$ $\left.S_{w i r r}\right) /\left(1-S_{w i r r}\right)$ and $k_{r n w}\left(S_{w i r r}\right)$ is the non-wetting relative permeability at the irreducible wetting saturation. Power law functions are typically used to describe relative permeability (Blunt, 2017; Picchi \& Battiato, 2019), and can be derived at limiting flow regimes directly from pore-scale physics. The macroscopic capillary pressure of the system during drainage can also described using a power-law function, introduced by Brooks \& Corey (Brooks \& Corey, 1964):

$$
P_{c}^{d}=P_{e}\left(S_{w}^{*}\right)^{\frac{-1}{\lambda}}
$$

where $P_{e}$ is the entry pressure, and $\lambda$ is the pore-size distribution factor.

\subsection{Representative elementary volumes}

Essential to the continuum framework is the concept of the representative elementary volume (REV). An REV is the volume around a material point in which the average of a microscopic quantity is independent to further incremental changes in volume (Bear, 1989). There is generally an upper bound in heterogeneous media due to macroscopic variations, and a lower bound when reaching the characteristic length scale of the microscale quantity (i.e. the pore/grain size when considering porosity).

There are different REV sizes for different properties, e.g., porosity, fluid saturations (Bear, 1989). These may also be transient in nature. The REV for porosity and absolute permeability has been studied extensively by (Arns et al., 2005; Hilfer \& Lemmer, 2015; Al-Raoush \& Papadopoulos, 2010; Razavi et al., 2007). In contrast, analysis of the REV for multi-phase flow properties, saturation, capillary pressure and relative permeability, has been limited by the difficulty in quantifying these properties at small spatial scales.

In this work, we use two approaches to analyse the REV for porosity, saturation, and capillary entry pressure. Firstly, we use a statistical moving average approach to identify an appropriate REV size (Costanza-Robinson et al., 2011; Al-Raoush \& Papadopoulos, 2010). A cubic volume is isotropically grown from the centre of the rock core, with averages obtained at each incremental step. The cube is then moved downstream by one 
voxel and the process repeated, sampling the pore space in an highly overlapping manner.

A second measure for the REV is determined from the spatial correlation of each property, derived through an empirical semi-variogram. Semi-variograms are often used in geostatistical field-scale modelling to represent the spatial correlation of geological structures (Jensen et al., 1996; Ringrose \& Bentley, 2015), but are increasingly used in the analysis of pore (AlRatrout et al., 2018) and core (Pini \& Benson, 2013) scale analysis of multiphase flow. The discrete semi-variogram $\xi(r)$ of a quantity $\psi$ can be formulated as (AlRatrout et al., 2018):

$$
\xi(r)=\frac{\sum_{j=1}^{N_{j}} \sum_{i=1}^{N_{i}} l_{i j}\left(\psi_{i}-\psi_{j}\right)^{2}}{2 \sigma^{2} \sum_{j=1}^{N_{j}} \sum_{i=1}^{N_{i}} l_{i j}}
$$

where $\sigma$ is the standard deviation of the property and $l_{i j}=1$ if $r-\epsilon<r_{i j}<r+\epsilon$ or 0 otherwise. $\epsilon$ is a tolerance used to bin the discrete distances, here set to half the minimum averaging size.

Macroscopic porosities and saturations are calculated directly using the micro-CT images with equation (1) at the associated averaging volumes. Macroscopic capillary entry pressures are then calculated using the capillary equilibrium assumption and the calculated average non-wetting saturations. Here, we first compute the average saturation for the whole core, and find $P_{c}$ using equation (5). For each smaller averaging volume, we then compute the averaged saturations, and compute a scaling factor for $P_{c}$ described in section 3.4, which gives an estimate for the entry pressure of that volume.

\subsection{Hysteresis}

Hysteresis in the macroscopic functions (capillary pressure and relative permeability) can stem from several pore-scale mechanisms which are not explicitly incorporated into the classical homogenization approach, namely: 1 - inkbottle effects during drainage and imbibition (Morrow, 1970; McClure et al., 2018), 2 - fluid connectivity (Berg et al., 2016) and 3 - wetting behaviour (Spiteri et al., 2008). It has been proposed that the capillary pressure function should incorporate the fluid-fluid interfacial area (Joekar-Niasar et al., 2007; Pyrak-Nolte et al., 2008; Porter et al., 2010) or a more detailed description of the geometric state of the fluids (McClure et al., 2018; Armstrong et al., 2018) to remove this hysteresis, and provide a unique macroscopic description of the capillary pressure through all displacement regimes.

Apparent hysteresis and flow rate rate dependency of macroscopic $P_{c}, k_{r \alpha}$ can also be manifestations of porous media heterogeneity, notably permeability and associated capillary pressure heterogeneity at the REV scale (Honarpour et al., 1995; Pini et al., 2012; Krause et al., 2013; Krause \& Benson, 2015; Reynolds \& Krevor, 2015; Jackson et al., 2018). When heterogeneity length scales approach the averaging scale, the derived $k_{r \alpha}$ and $P_{c}$ functions can display flow rate dependence and hysteresis even if the intrinsic, homogeneous function does not. This was recently demonstrated in Jackson and Krevor (2019) whereby the intrinsic water relative permeability did not exhibit hysteresis, but with the inclusion of structural heterogeneity impacts, the equivalent core-averaged water relative permeability was found to exhibit hysteresis.

Under conditions where the multiphase extension of Darcy's law is valid, we can make use of current macroscopic modelling approaches to evaluate flow experiments where fluid morphology is imaged at the pore scale. This allow us to asses the validity and limits of current continuum scale models. Using pore-scale imagery we parameterise the Land 
continuum hysteresis model which is based on a conceptual picture of the pore-scale connectivity of the non-wetting fluid phase.

Hysteresis in the imbibition non-wetting relative permeability can be parametrised using the initial-residual saturation relationship, e.g. Land (1968); Carlson (1981); Jerauld (1997); Spiteri et al. (2008). For water wet rocks, the Land model has been found to provide a good match to many experimental datasets (Spiteri et al., 2008). The Land model relationship between the initial non-wetting saturation $S_{n w, i}$ (i.e. the turning point at the end of drainage) and the residual saturation $S_{n w, r}$ (i.e. the end-point of imbibition) is described by:

$$
S_{n w, r}=\frac{S_{n w, i}}{1+C_{l} S_{n w, i}}
$$

where $C_{l}$ is the Land trapping parameter. Applying the Land model at different saturation states in the imbibition displacement process yields a relationship for the connected (mobile) non-wetting saturation, as a function of the total non-wetting saturation $S_{n w}$ and trapping characteristics:

$$
S_{n w, c}=\frac{1}{2}\left(-\Delta+\sqrt{\Delta^{2}-4 \Delta / C_{l}}\right), \quad \Delta=S_{n w, r}\left(S_{n w, i}\right)-S_{n w}
$$

The primary imbibition relative permeability $k_{r n w}^{i}$ can then be calculated based on the equivalent drainage relative permeability $k_{r n w}^{d}$ at the connected non-wetting saturation $S_{n w, c}$ (Land, 1968):

$$
k_{r n w}^{i}\left(S_{n w}\right)=k_{r n w}^{d}\left(S_{n w, c}\right)
$$

For intermediate scanning relative permeabilities $k_{r n w}^{s}$ between the primary drainage and primary imbibition curves, the Killough interpolation method is used (Killough, 1976.), see the SI for full details.

For wetting relative permeability, there is less dependence on the trapping of the non-wetting phase during imbibition in water-wet media (Oak, 1980), although there have been examples of a slight raising of the wetting relative permeability during imbibition as is seen in the experiments here (see also C. Reynolds (2016); Shi et al. (2017); Akbarabadi and Piri (2012)). This is caused primarily by the wetting fluid occupying larger pores during imbibition than in drainage (Blunt, 2017). This is characterised by using a different exponent in the power-law relative permeability expressions for imbibition and using a similar Killough scanning method as per the non-wetting case (see the SI).

The imbibition capillary pressure $P_{c}^{i}$ is formulated in a similar fashion to the drainage case with a modification of the Brooks-Corey model for drainage, using similar notions as the non-wetting relative permeability during imbibition (Pini \& Benson, 2017):

$$
P_{c}^{i}=P_{c i}\left(\left(1-S_{n w, c}^{*}\right)^{\frac{-1}{\lambda}}-1\right)+P_{s}
$$

Here, $P_{s}$ is the imbibition snap-off pressure, which is positive for water-wet media, negative for oil-wet media, and sometimes very close to zero for mixed wet media (Lin et al., 2018, 2019). $P_{c i}$ can be found by equating the capillary pressures during drainage and imbibition at the turning point. Due to the complexity of tracking $P_{c i}$ with subsequent loops, we use an interpolation method to scan between the primary drainage and imbibition curves, again in the manner described by (Killough, 1976.) (see the SI for equations).

\subsection{Heterogeneity}

The final part of the continuum modelling considers the heterogeneity in the system arising from REV level variations in porosity, permeability and capillary pressure 
characteristics. Fluid properties remain approximately constant throughout the experiment (the pore pressure in the experimental core is $1.5 \mathrm{MPa} \pm 0.015 \mathrm{MPa}$ and temperature is $30^{\circ} \mathrm{C} \pm 0.5^{\circ} \mathrm{C}$, leading to variations in viscosity and density of decane of $<0.8 \%$ and $<0.06 \%$ respectively; there is similar uncertainty in the brine properties).

Heterogeneities in sandstones are a consequence of varying pore structure throughout the rock caused by variations in sedimentary deposition and diagentic processes (Corbett et al., 1992). Porosity can be found directly from the 3D micro-CT image, arithmetically averaged to an appropriate REV. For the permeability, we use the measured core average permeability. While the permeability will vary at the REV scale in the $3 \mathrm{D}$ core (Krause et al., 2013), at the low microscopic and macroscopic capillary numbers in the present experiment, permeability variations at the REV scale have little impact on fluid distributions (Jackson et al., 2018). More prominent is heterogeneity in the capillary pressure characteristic (S. Krevor et al., 2015; Jackson et al., 2018; Reynolds \& Krevor, 2015).

In order to characterise capillary pressure heterogeneities, we use the imaged fluid saturation heterogeneity as a signal (Chaouche et al., 1993, 1994) coupled with an average capillary pressure - saturation function (Pini et al., 2012). We scale the capillary pressure function using the following expression:

$$
P_{e}=\frac{\bar{P}_{c}\left(\bar{S}_{w}\right)}{\bar{P}_{c}\left(S_{w i}\right)} \cdot \bar{P}_{e}
$$

where $P_{e}, \bar{P}_{c}\left(\bar{S}_{w}\right), \bar{P}_{c}\left(S_{w i}\right)$ and $\bar{P}_{e}$ are the REV entry pressure, the capillary pressure at the core averaged saturation $\bar{S}_{w}$, the capillary pressure at the REV saturation $S_{w i}$, and the average capillary entry pressure. The average capillary pressure can be obtained from the model fit (i.e. Brooks-Corey (Brooks \& Corey, 1964)) to experimental porous plate, centrifuge, or mercury intrusion capillary pressure data. Here, it is constrained by the porous plate data of (Raeesi et al., 2014) and our curvature based $P_{c}$ measurements (see the SI)

The term $\frac{\bar{P}_{c}\left(\bar{S}_{w}\right)}{\bar{P}_{c}\left(S_{w i}\right)}$ in equation (11) is often referred to as the scaling factor (Jackson et al., 2018). The scaling assumes that the capillary pressure at the REV scale is at local equilibrium. The capillary pressure heterogeneity can also be found using multiple fractional flows (Krause et al., 2013; Jackson et al., 2018; Jackson \& Krevor, 2019), however, here we find that the saturation in the first fractional flow gives the best signal for the capillary pressure, and it is closer to capillary equilibrium than other fractional flows, making the scaling most effective. We choose not to perform any iterative calibration (Jackson et al., 2018), so as to keep the model as simple as possible. The same scaling factor can be used during both drainage and imbibition, to modify the base capillary pressure functions at a specific voxel (Pini \& Benson, 2017).

\subsection{Numerical modelling}

With the well-posed continuum framework we can also numerically simulate the multiphase flow experiments. Numerically, we solve equations (2) - (3) on a 3D grid representation of the cores using the fully implicit, isothermal immiscible multiphase porous media flow simulator CMG IMEX ${ }^{\mathrm{TM}}$, in an approach developed previously by the authors (Jackson et al., 2018; Jackson \& Krevor, 2019).

Full details of the numerical modelling and verification are available in the SI and previous papers. In summary, the model is populated with the average capillary pressure functions described in sections 3.1 and 3.3, with parameters obtained from the experimental observations (see the SI). Porosity and end-point capillary pressures are assigned to each 'REV voxel' in the 3D numerical model, along with core averaged permeability to represent the sub-core scale heterogeneity of the system. The intrinsic, rate independent relative permeability function is prescribed to every location in the core (see 
section 4.5) for both drainage and imbibition. The core-averaged trapping parameters $\left(C_{l}\right)$ are used with the Land trapping model to define the connectivity evolution and hysteretic relative permeability and capillary functions. Constant fluid properties and temperatures are used throughout the simulations.

\section{Results and discussion}

Here we present experimental observations and evaluate predictions from the continuum scale modelling approaches. The results focus on the ability of the continuum models to capture hysteresis and the impacts of heterogeneity. We first evaluate the REV of the system, followed by fluid connectivity and hysteresis. We end with 3D numerical model predictions of fluid saturation, core-averaged relative permeability, and trapping during drainage and imbibition.

\subsection{Representative elementary volumes}

Figure 3 shows the result of REV calculations in the Bentheimer samples. Extra information and specific moving averages for each cube realization are available in the supporting information. The standard deviation for all 350 unique cube realizations for different properties are displayed in Figure 3a, showing the transition as the volume approaches the largest averaging size with side length $7.5 \mathrm{~mm}$. There is a difference in REV length scale between the porosity, entry pressure, and saturation. Above $2 \mathrm{~mm}$ and $4 \mathrm{~mm}$ side length the porosity and entry pressure variation drops below $5 \%$ and $1 \%$ respectively, whereas the saturation still shows significant variation $>10 \%$. Although the capillary pressure is calculated using the saturation as a scaling, the large saturation fluctuations are explained by relatively small fluctuations in capillary pressure characteristics, which has been observed in several experiments (Reynolds \& Krevor, 2015; Reynolds et al., 2018).

The REV of saturation varies with the flow regime; we plot the estimated REV for saturation at a $5 \%$ property variance (i.e. found through Figure 3a) for each of the fractional flows in Figure 3b. In Figure 3b, the saturation REV is maximised at low fractional flows, especially during drainage. Here the capillary number is low and small variations in capillary pressure characteristic can have large impacts on fluid distribution. The REV side length at $5 \%$ variation for saturation ranges between 1.5 and $5.4 \mathrm{~mm}\left(3.4 \mathrm{~mm}^{3}\right.$ and $157.5 \mathrm{~mm}^{3}$ volume) for both samples, in comparison to $\approx 1.25 \mathrm{~mm}\left(2 \mathrm{~mm}^{3}\right.$ volume) for porosity and capillary pressure.

The spatial correlation $\xi(r)$ of the porosity and capillary pressure are shown in Figures $3 \mathrm{c}$ and $3 \mathrm{~d}$ respectively, for 7 distinct cube volumes sampled along the length of the core. The spatial correlation highlights the local variance in the properties; it represents a lower bound on the REV, which is generally much larger to ensure separation of scales (Nordahl \& Ringrose, 2008). The lag distance to the sill, or plateau, in $\xi(r)$ indicates the point at which the property variance reaches the variance at the largest averaging volume considered. The sill distance is clearly visible at $\approx 0.6 \mathrm{~mm}$ for porosity and $\approx 1.25 \mathrm{~mm}$ for capillary pressure. Note the scaled spatial correlation of the saturation is identical to the capillary pressure in Figure $3 \mathrm{~d}$. The plots also highlight the large micro-scale variance by the large 'nugget' or offset in the $\xi(r)$ value at the smallest volume used of 350 $\mu \mathrm{m}$. $\xi(r)$ computations at volumes smaller than $350 \mu \mathrm{m}$ result in large computational times, but result in $\xi(r)$ approaching a small nugget near 0 . There is also macro-scale, periodic heterogeneity indicated by the periodic variation at large lag distances, indicative of inhomogeneity at large scales. The figure also shows section $5(x / L=5 / 7)$ of core 2 , where the tight, low porosity/permeability exists (see Figure 2). Here the sill distance does not occur until at least $4 \mathrm{~mm}$, indicating the spatial structure of the low porosity band in the sample. Note, here we consider isotropic correlations, which are generally observed in the core at these scales, further consideration of anisotropy could resolve the seemingly large correlation length of the banded feature (Yoon \& Dewers, 2013). 


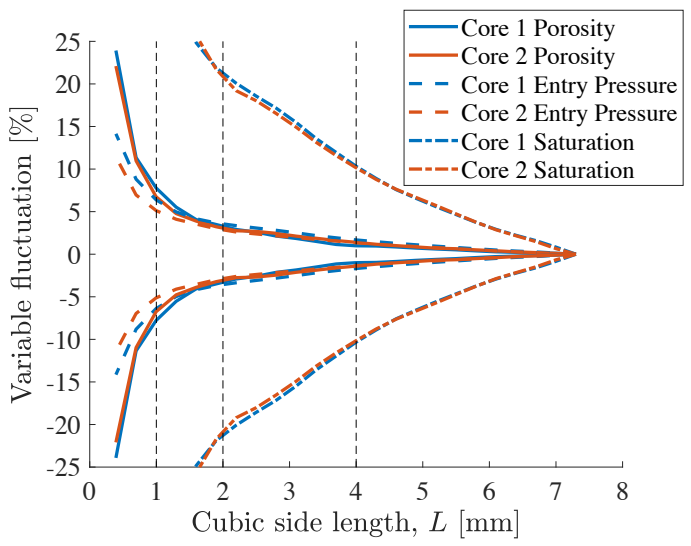

(a)

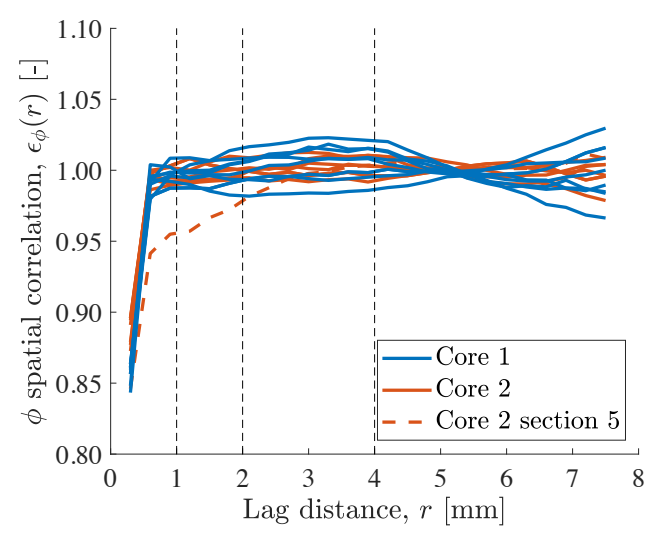

(c)

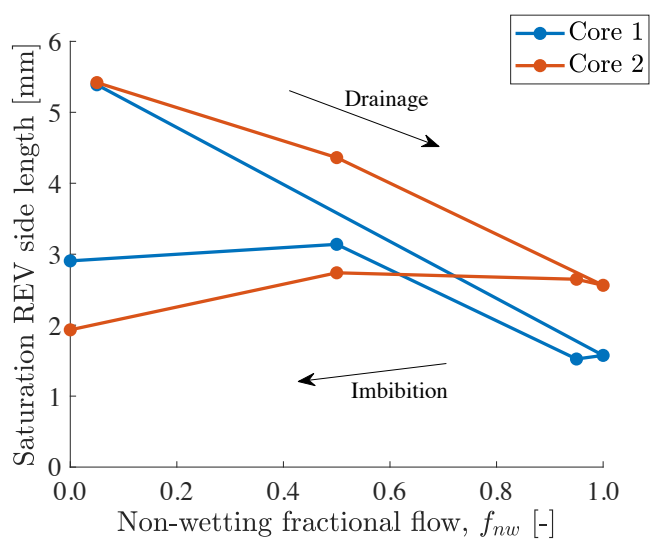

(b)

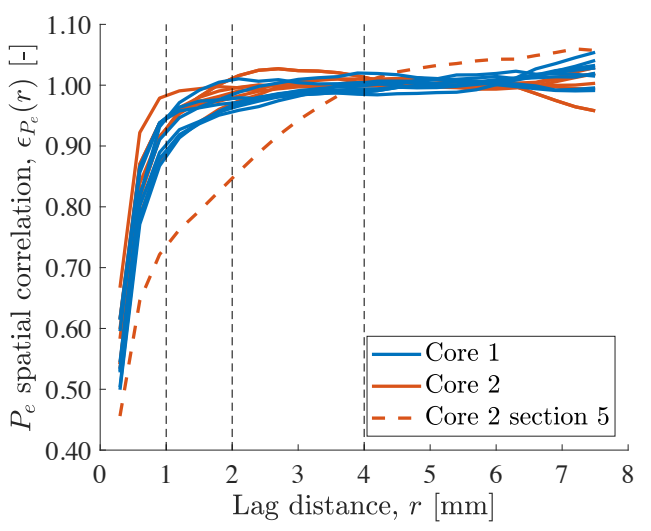

(d)

Figure 3. REV analysis for porosity, entry pressure and saturation. (a) Core 1 and 2 porosity, entry pressure and $f_{n w}=0.05 \mathrm{nw}$ saturation fluctuation with averaging volume. The fluctuation represents the \pm standard deviation of 350 moving average realizations, comparing to that from the largest averaging volume with side length of $7.5 \mathrm{~mm}$. (b) Saturation REV estimate at $5 \%$ variable fluctuation for different fractional flows. (c) Porosity spatial correlation with lag distance. (d) Capillary entry pressure spatial correlation with lag distance. 
Previous studies on Bentheimer sandstones have identified an REV for porosity of $>1.75 \mathrm{~mm}$ (Halisch, 2013) and saturation of $>3.5 \mathrm{~mm}$ (Herring et al., 2013), largely in line with the results here. Using similar approaches, (Costanza-Robinson et al., 2011) identified porosity REVs in the range 0.05 to $8 \mathrm{~mm}^{3}$ and saturation REVs up to $50 \mathrm{~mm}^{3}$ in a selection of glass beads and sintered silica samples. (Fernandes et al., 2012) calculated porosity REVs $>1.4 \mathrm{~mm}$ in a variety of sandstone and siltstone reservoir samples. Further, in (Hilfer \& Lemmer, 2015), the porosity REV for Fontainbleu sandstone was identified as at least $10 \mathrm{~mm}$. The large range of reported REVs for similar sandstone/silica based systems stems from sample variations (i.e. average pore size distributions and permeability), and that from inherent user-bias, i.e. arbitrarily selecting the REV as the point at which the property variation drops below a certain threshold. To remove sample variations, REVs can be described non-dimensionally as $V_{R E V}=V / \mathrm{d} 50^{3}$, where $\mathrm{d} 50$ is the average grain diameter at which $50 \%$ of all grains have a smaller diameter (Al-Raoush \& Papadopoulos, 2010). Using this approach, (Al-Raoush \& Papadopoulos, 2010) identified the non-dimensional REV of several sand pack samples as $V_{R E V}=2 \times 10^{4}$, i.e. a cubic side length $d_{R E V}$ of 27 grains. This is similar to that found by (Razavi et al., $2007)$ in sand-packs ranging from $d_{R E V}=5.4$ - 15.4. In the samples here, with an average d50 $\approx 80 \mu \mathrm{m}$ (Peksa et al., 2015 ), $d_{R E V}$ is $\approx 15.6$ for porosity and capillary entry pressure and $\approx 18.8-67.5$ for saturation at $5 \%$ property variation.

To remove user-bias, and arbitrariness in REV determination, the choice of REV has to be intrinsically linked to an associated systematic uncertainty, which is evaluated in the sense of model predictions. Comparing model predictions to observations, and linking this with the uncertainty in the REV provides a robust way of estimating errors in modelling. Ensuring that that the REV is significantly larger than the correlation length of the properties (which is scaled to the property system variance, and hence does not necessarily indicate an REV) ensures that resulting models and simulations will be independent of the averaging size (Nordahl \& Ringrose, 2008). Here, we choose the $5 \%$ cutoff in property variation for our REV determination, which results in an REV with a minimum side length of $1.25 \mathrm{~mm}$. Throughout the rest of the article, we use an REV side length $\approx 1.9 \mathrm{~mm}$ for $3 \mathrm{D}$ computations (the specific size varies between the cores, and is chosen to easily average the CT images with defined integer dimensions - for core 1 , the size is $1.95 \times 1.95 \times 1.8 \mathrm{~mm}$, core 2 is $1.9 \times 1.9 \times 1.9 \mathrm{~mm}$ ) noting the relative uncertainty of at least $5 \%$ in the saturation at these scales. The saturation variation can be larger at different fractional flows, which is considered in the analysis. This REV size provides a balance of spatial representation inside the core (i.e. we have a statistically significant number of 'REV sized voxels') and precision when assessing 3D models. In the supporting information, further detailed REV plots are included for porosity, saturation and capillary pressure, along with simulation results presented at various REV sizes in SI Figure 16.

\subsection{Fluid saturation and connectivity}

Having determined appropriate REVs for the system, we now consider the experimental fluid connectivity during the steady-state experiments. Figure 4 shows the connected and disconnected macroscopic saturations for the two Bentheimer cores. A crosssection image of Core 1 is also shown with pore-scale connected and disconnected fluid distributions which are used in the macroscopic calculations. The error bars in the macroscopic saturation for imbibition fractional flow $f_{n w}=0.5$ (with significant connected/disconnected saturations) indicate the segmentation uncertainty, generated by varying the greyscale threshold values for the non-wetting phase by \pm 200 units, i.e. $\pm 3.3 \%$ of the original greyscale values. The uncertainties in Figure 4 (and in SI table 1) highlight the robustness of the segmentation methodology; saturations and connectivity trends are relatively insensitive to the choice of thresholding limits used in the watershed segmentation. 


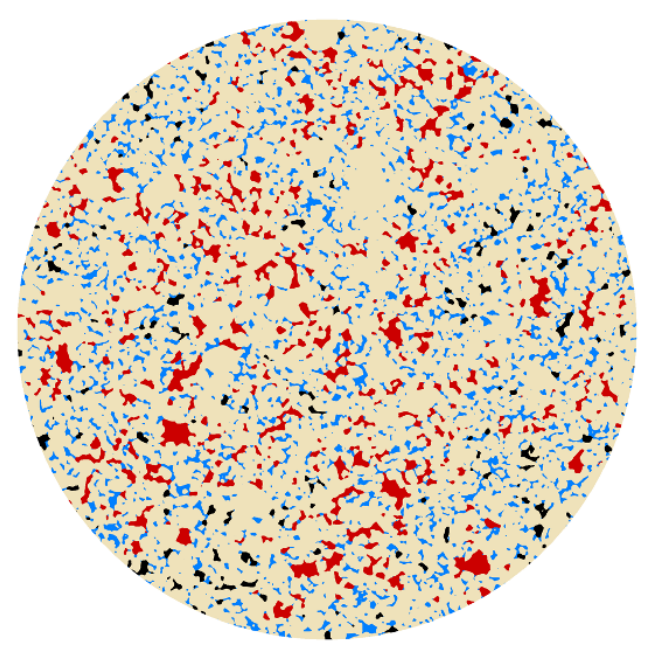

(a)

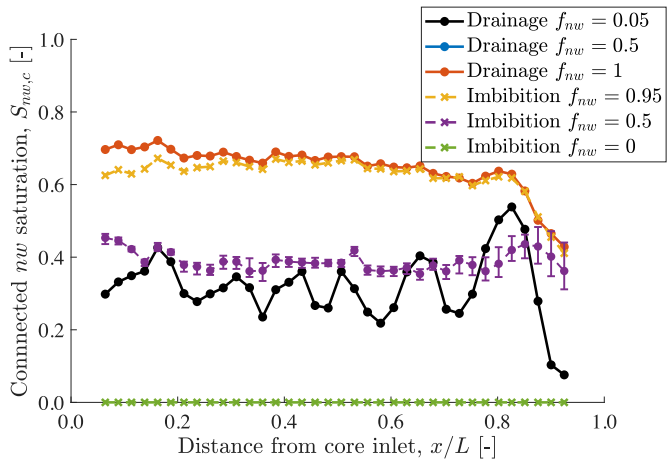

(b)

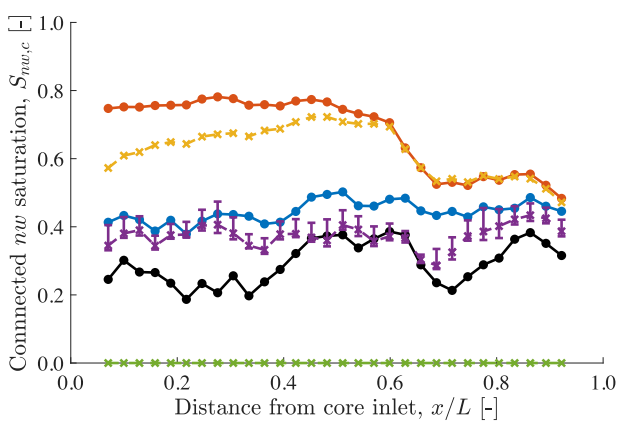

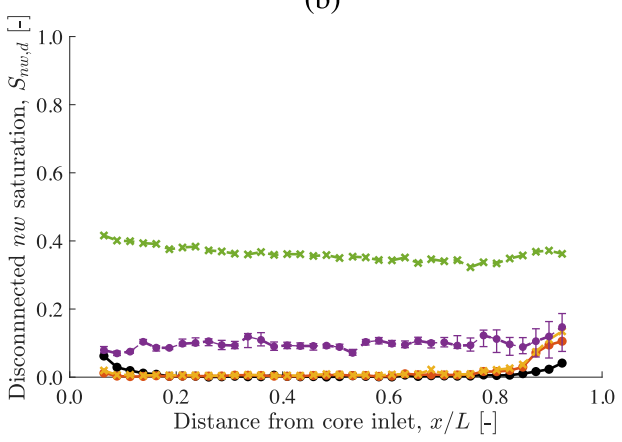

(d)

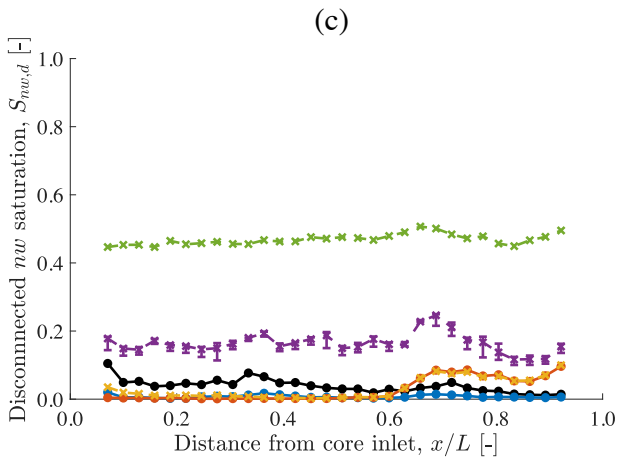

(e)

Figure 4. (a) Central cross-section $(6 \mu \mathrm{m}$ thick, $x / L=0.5)$ of core 1 showing segmented pore-space (off-white), brine (blue), connected oil (red) and disconnected oil (black) for imbibition $f_{n w}=0.5$. In this image, $S_{n w, c}=0.412, S_{n w, d}=0.096$. (b-e) Experimental slice average non-wetting saturations during drainage and imbibition. (b), (d) Connected and disconnected non-wetting saturations for core 1 respectively. (c), (e) Connected and disconnected non-wetting saturations for core 2 respectively. Error bars for imbibition $f_{n w}=0.5$ represent segmentation sensitivities. Slice thickness's are $1.8 \mathrm{~mm}$ and $1.9 \mathrm{~mm}$ for core 1 and 2 respectively.

In Figure 4, during drainage at low $f_{n w}$, the non-wetting phase saturations are highly heterogeneous, showing large fluctuations of $\pm 22.2 \%$ and $\pm 15.7 \%$ around the mean for core 1 and core 2 respectively. The fluctuation is much larger than that apparent in the 
porosity profiles in Figure 2, indicating the role of capillary pressure heterogeneity. Indeed, the slice volumes here are $>170 \mathrm{~mm}^{3}$, above the previously identified REV for saturation. As the fractional flow of non-wetting phase increases, the saturation heterogeneity is reduced to $\pm 6.1 \%$ and $\pm 10.5 \%$, impacted increasingly by the outlet end effect where $P_{c} \rightarrow 0$.

During the primary drainage process, there is a small, but apparent volume of disconnected phase, displayed in Figure 4d, e. Low non-wetting phase saturations $S_{n w} \approx$ 0.1 can still contain several disconnected ganglia and contribute to variations in fluid conductivity through the sample. In the more homogeneous core 1, the trapping occurs mainly at the core inlet and outlet and is possibly due to intermittent connectivity caused by the fluid entering and exiting the core and segmentation connectivity artefacts.

The disconnected $S_{n w, d}$ phase is much more prominent during drainage in the heterogeneous sample, core 2 . This manifests itself around the low porosity/permeability barrier at $x / L \approx 0.7$, where there is a significant jump in disconnected saturation. Several segmentation thresholds were tested to ensure the disconnected phase is real. Localised, high entry pressure regions are known to cause build-ups of $S_{n w}$ during drainage (Krevor et al., 2011), but here we also see an increase in the localised trapping (due to snap-off of non-wetting phase) during drainage.

Trapping during a primary drainage process has been recently reported by (Herring et al., 2018), through image analysis of micro X-Ray CT experiments. These observations could not be described by a classical invasion-percolation process. The mechanism was attributed largely to 'Roof snap-off' during the low capillary number flow regime, which was initially observed by (Andrew et al., 2015) during time resolved, unsteadystate synchrotron experiments. This process relies on pore space heterogeneity and sufficient aspect ratio between adjacent pore bodies and throats. While this process could be occurring during this experiment, and account for some of the trapping seen during drainage, a more significant mechanism is likely that caused by the pore space heterogeneity itself. At high drainage fractional flows of non-wetting phase, water is displayed downstream and initially trapped ganglia in the upstream section of the core are reconnected. With a reduction in the pore and throat sizes downstream in the low porosity band, the displaced water starts to fill these areas preferentially, and can eventually cause the snap-off of non-wetting phase. A contrast in pore size distributions between adjacent regions necessarily enhances snap off during drainage, due to preferential filling of water in the small pore size region. This mechanism occurs simultaneously with the buildup of connected non-wetting phase behind the low entry pressure region, resulting in a highly heterogeneous saturation profile. This mechanism is likely weaker in the more homogeneous core, since the pore-size distribution is more continuous; displaced water fills the core more homogeneously and Roof snap-off occurs preferentially.

\subsection{Pore-filling}

The pore filling sequence of the connected and disconnected non-wetting phase are shown in Figure 5 for the whole core (detailed pore-filling histograms are shown in SI Figure 11, for 4 sections of the core). During drainage near the percolation threshold, there is a large range of pore sizes invaded by the connected non-wetting phase, highlighting the difference between pore availability and accessibility (Lenormand et al., 1983; Ruppert et al., 2013). The initial invasion percolation process is dominated by piston like displacement and Haines jump pore filling mechanisms (Haines, 1930; Lenormand et al., 1983; Berg et al., 2013), followed by slower corner-flow drainage at low water saturations (Tuller \& Or, 2001). Based on macroscopic capillary pressures it would be expected that the largest pores in the system would be invaded by the non-wetting phase first, however in Figure 5a, b there remains a significant proportion of the largest pores unfilled at low fractional flows. The pore space topology renders some of these large pores 


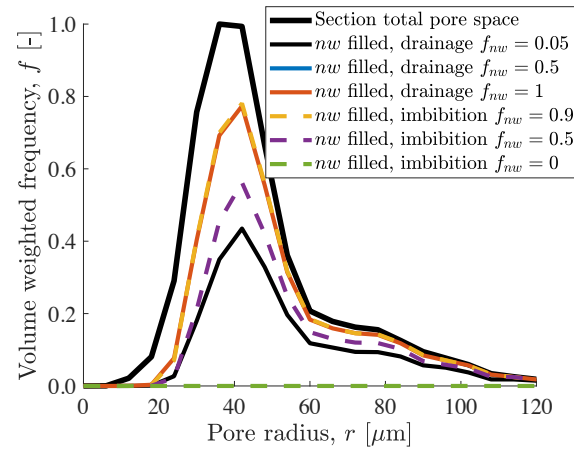

(a)

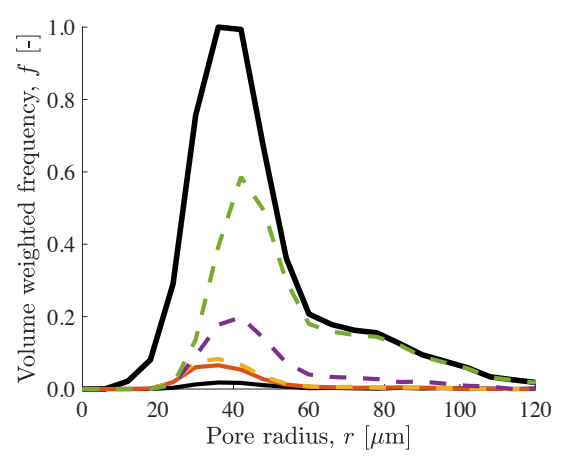

(c)

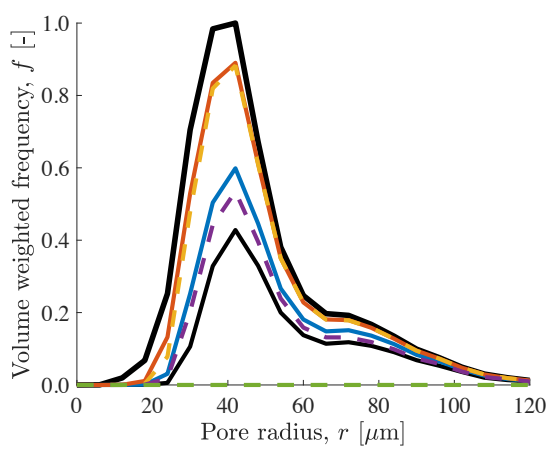

(b)

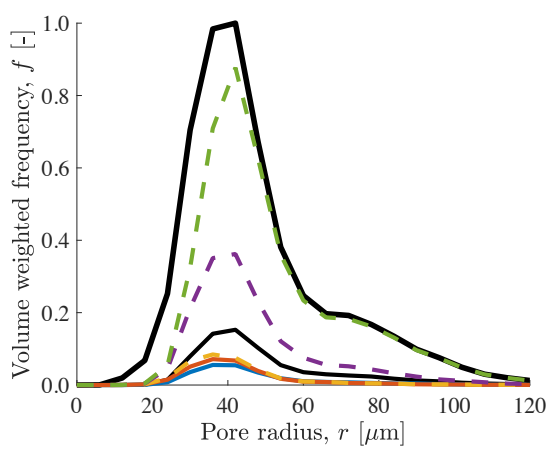

(d)

Figure 5. Pore volume weighted histograms showing the non-wetting pore filling sequence during drainage and imbibition, for connected and disconnected non-wetting phases. (a), (c) Connected and disconnected non-wetting filling for core 1 respectively. (b), (d) Connected and disconnected non-wetting filling for core 2 respectively.

inaccessible; they are surrounded by regions of high-entry pressure. Increasing the capillary pressure as $f_{n w}$ is raised increases their accessibility, and at $f_{n w}=1$ almost all the largest pores in the system are filled with connected non-wetting phase.

The disconnected non-wetting phase first emerges in the smallest pores in the system, highlighted in Figures 5c, d. Water preferentially swells from the corners of the throats in these regions and eventually re-connects to cause non-wetting phase snap-off in I1 and I2 events (Lenormand et al., 1983); this is true during both primary drainage and imbibition processes. At the end of imbibition, $f_{n w}=0$, the non-wetting phase is completely disconnected, occupying only the largest pores in the system. The saturations during imbibition are much more homogeneous than those during drainage for the same fractional flow $\left(f_{n w}=0.5\right)$ and similar average saturations $\left(S_{n w} \approx 0.35-0.45\right)$ shown in Figure 4. The favourable free-energy change when displacing the non-wetting phase means there is less energy required for the same volume change during imbibition (Morrow, 1970; McPhee et al., 2015). The capillary pressure required during imbibition to create similar volumetric fluid configurations is lower than that during drainage; this also reduces the overall magnitude of capillary pressure gradients in the rock. With a reduction in capillary pressure gradients, the saturation gradients also reduce, creating a more homogeneous profile through the core. 


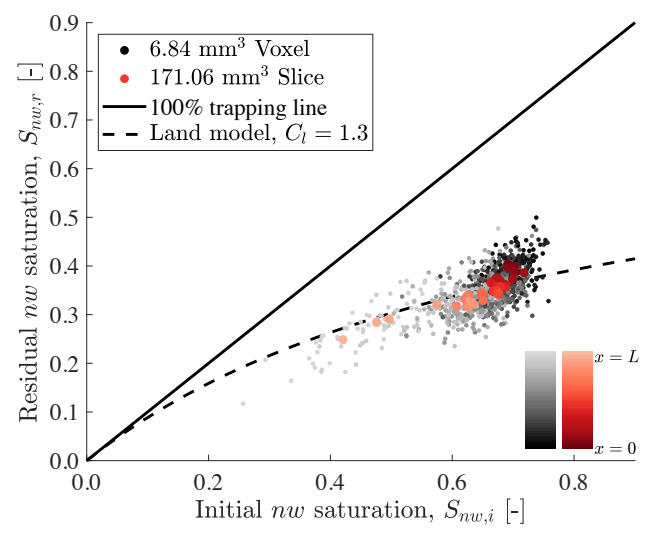

(a)

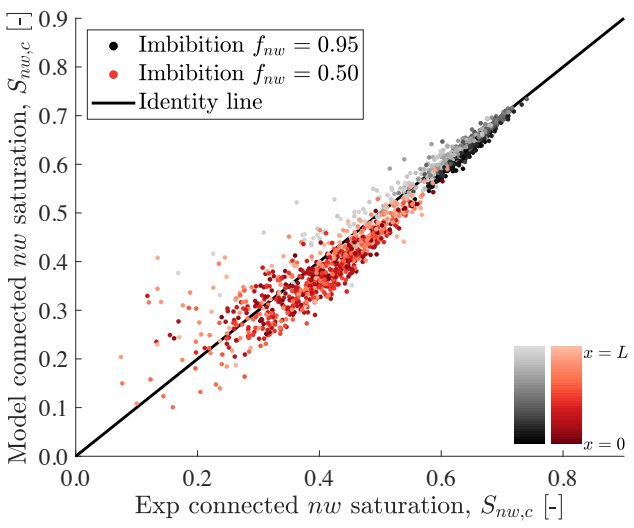

(c)

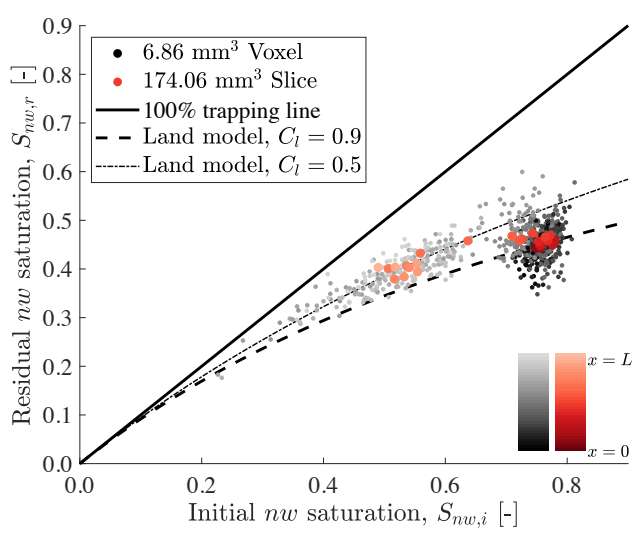

(b)

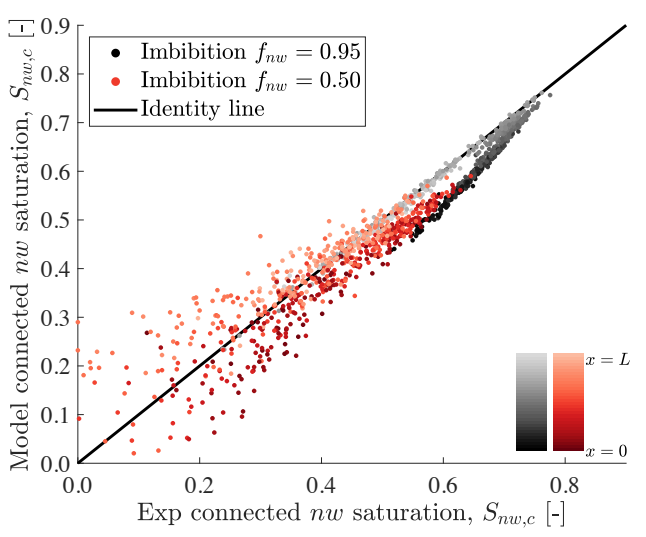

(d)

Figure 6. Experimental trapping characteristics at different resolutions. (a), (b) Initialresidual plots for core 1 and 2 respectively, showing trapping measurements at different resolutions with Land parametrisations.(c), (d) Trapping model predictions at different fractional flows compared to the experimental measurements.

\subsection{Continuum-scale trapping and fluid connectivity}

The trapping characteristics of the two cores are displayed in the initial - residual plot in Figure 6a, b. The initial saturations here are the connected non-wetting phase at the end of drainage, whilst the residuals are the disconnected non-wetting phase at the end of imbibition minus any disconnected non-wetting phase at the end of drainage. This is key to accurately track the connected phase evolution, and not overestimate the level of trapping by considering already trapped ganglia from earlier in the displacement process.

A proportion of the spread in the voxel data is typically explained with the random error in the saturation measurement from X-Ray CT scanning (Pini et al., 2012; Jackson et al., 2018; Ni et al., 2019). Recently in (Ni et al., 2019) over a wide range sandstone samples this was as high as $7.9-9.8 \%$ for $10 \mathrm{~mm}^{3}$ voxels, which was reduced to $0.22-$ $0.3 \%$ for slice averages using a differential imaging medical CT scanner setup. In the microCT experiment here, the segmentation sensitivity is linearly propagated through the volume averaging, meaning that large voxels have the same intrinsic uncertainty in saturation as small voxels due to segmentation uncertainty. At $f_{n w}=1$ during drainage 
and $f_{n w}=0$ during imbibition, the non-wetting phase is almost completely connected and completely disconnected respectively, meaning saturation uncertainty is in the range $1.3 \%-4.1 \%$ (see SI Table 1), smaller than the scatter observed in the plot. Instead, the scatter stems from the inherent REV of the saturation; estimated variations at the averaging voxel size are 5-10\%, see Figure $3 \mathrm{a}, \mathrm{b}$, and account for most of the spread in data around the intrinsic trapping The slice averages are taken above the saturation REV and capture the intrinsic trapping, which is parametrised well in this case by a Land model (Land, 1968) for a relatively homogeneous water-wet sample (core 1).

In the more heterogeneous core 2 in Figure 6, we see evidence of capillary heterogeneity trapping, i.e. enhanced trapping above that predicted by snap-off processes in a homogeneous core (Krevor et al., 2011). The slice average saturations show a similar trend to that at the voxel level, with an increase in the amount of trapping towards the low porosity section. The variation in trapping is larger than the uncertainty in saturation REV, and can be associated with REV scale heterogeneities in capillary pressure. Through knowledge of the REV of the system, and the resulting uncertainty, the underlying trapping process can be elucidated.

The general amount of trapping in core 2 has been increased over core 1, even though they exhibit very similar pore-size distributions through the majority of the core (see Figure 5). The low porosity band in core 2 has raised the initial non-wetting saturation compared to core 1, through build-up of non-wetting phase behind low entry pressure regions, and has also trapped it at higher non-wetting saturations following imbibition. Water preferentially imbibes the small pores/throats in the downsytrean region, snapping off the non-wetting phase at higher saturations than in upstream regions.

This kind of enhanced trapping has been observed in larger scale experiments in heterogeneous media (Krevor et al., 2011; Reynolds et al., 2018; Trevisan et al., 2017), but here the results are much more explicit. Previous experiments rely on bulk, total saturations to calculate the initial-residual relationships, assuming that all the non-wetting phase at the end of drainage is connected, and it is completely disconnected at the end of imbibition. We have shown that for heterogeneous media this is not always true, trapping can play a significant role during drainage, altering the 'initial' saturation. Indeed, using the initial-residual approach based on total saturations for the experiments here over-predicts the level of trapping in the core significantly, since some fluid is already trapping at the end of drainage.

Further from the initial-residual analysis, the connected saturations can be tracked throughout the imbibition process at different fractional flows. Using equation (8), we can calculate the connected non-wetting phase saturation at any stage of imbibition based on the parametrisation of the initial - residual saturations. Here we use the Land model parameterisation, but this can be any parametrisation of the initial-residual data, e.g. Carlson (1981); Spiteri et al. (2008). We compare the predictions of non-wetting fluid phase connectivity from the Land model to the directly imaged fluid saturations in Figure $6 \mathrm{c}$ and $\mathrm{d}$. In core 1 , the connected saturation evolution is well predicted by the Land model for both the $f_{n w}=0.95$ and 0.5 flows, showing little scatter around the identity line. The inherent assumptions in the connected phase evolution are met in the waterwet homogeneous core, whereby with sufficient initial-residual data the entire mobile evolution can be predicted using the same functional form applied at different 'initial' states.

In core 2, Figure $6 \mathrm{~d}$, the model is parametrised with the lower Land coefficient $C_{l}=$ 0.9. At the first imbibition fractional flow $f_{n w}=0.95$, many of the voxels in the low porosity region are completely disconnected, so the mobile saturation prediction is very good, however for upstream points the model under-predicts the connected saturation. Based on the total saturation, the disconnected saturation predicted from the Land trapping model is roughly $5-10 \%$ higher than in seen in the experiments, Figure 4e. The high entry pressure region downstream causes a build-up of non-wetting phase in the upstream 
region, whilst also increasing the phase connectivity; local heterogeneity trapping. This is prominent at the low water fractional flow where the capillary pressure is high. In subsequent imbibition fractional flows the connected saturation is again well predicted by the model as the capillary pressure drops, saturations become more homogeneous and snap-off trapping dominates, with the larger spread in points for core 2 indicative of the more heterogeneous core.

Using the simple trapping parametrisation above, the evolution of the connected phase can be predicted and the relative competition of pore-scale displacement mechanisms considered. For homogeneous media, a suitable trapping parametrisation based on the initial-residual relationship seems sufficient to describe the connectivity evolution through multiple imbibition states, validating the conceptual model of trapping at the continuum scale. However, it is clear that heterogeneity manifesting from the spatial variation in pore-size distribution within a core can completely alter the level of trapping, and the imbibition displacement mechanisms that occur. The temporal evolution of trapping can follow different mechanisms, which are not generally accounted for in macroscopic, continuum scale models based on the initial-residual relationship. These variations have to be incorporated into models through information about heterogeneity in $3 \mathrm{D}$.

\subsection{Hysteresis and heterogeneity}

Further to the experimental (and analytical model) results, we now numerically model the observed displacement process at the continuum scale in $3 \mathrm{D}$, using the model parametrisation in section 3.5. We directly compare the simulated saturations (connected, disconnected) and predicted core-average relative permeabilities to those measured experimentally. Tabulated experimental and simulated data, along with associated uncertainties are displayed in the SI, and will be used throughout the discussion herein. Throughout, we compare continuum features centred on REV voxels, slices and the whole core, with sizes equivalent to those used previously.

Core length profiles and 3D maps of the simulated and experimental saturations are shown in Figure 7 . In core 1, the heterogeneous, near periodic saturation profile is well captured at both low and high non-wetting fractional flows. Using the low fractional flow saturation to scale the capillary entry pressure in the core has resulting in a strong prediction across the fractional flow regime. Without this scaling, even with strong permeability contrasts the saturation profile would be homogeneous. For core 2, the saturation heterogeneity is somewhat under-predicted. In this experiment, the higher differential pressure renders the displacement closer to the viscous limit, and hence scaling the entry pressure with the non-wetting saturation is less effective. This is apparent also in the proximity of the equivalent relative permeabilities to the intrinsic curves in Figure 8. In these plots, the voxel and slice volumes are much larger than the REV, and hence mismatches between the simulation and experimental saturations are largely due to under-prediction of the capillary heterogeneity in the system, as opposed to the choice of averaging scale.

In the experiments, we also observe a significant proportion of disconnected nonwetting phase during drainage, which is not predicted by the model, even with hysteretic relative permeability and capillary pressure functions. The simulated displacement occurs in a macroscopic invasion-percolation process in order of entry pressure (Nooruddin \& Blunt, 2017), with all voxels increasing monotonically in non-wetting saturation once invaded. It is possible that the disconnected non-wetting phase aids the localised saturation build-up of the connected phase, increasing the saturation heterogeneity in the system, something that should be considered in future model developments.

In Figures 7e, f we observe the impact of hysteresis; the saturation heterogeneity in the system has been significantly reduced compared to drainage (see Figure 7a,b) an 


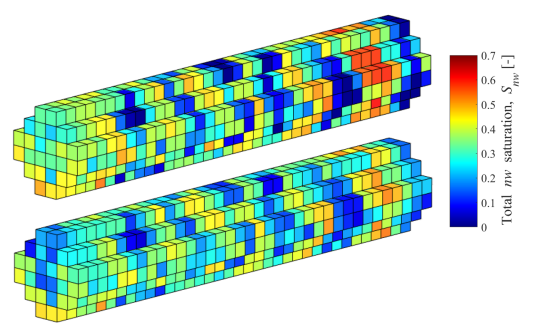

(a)

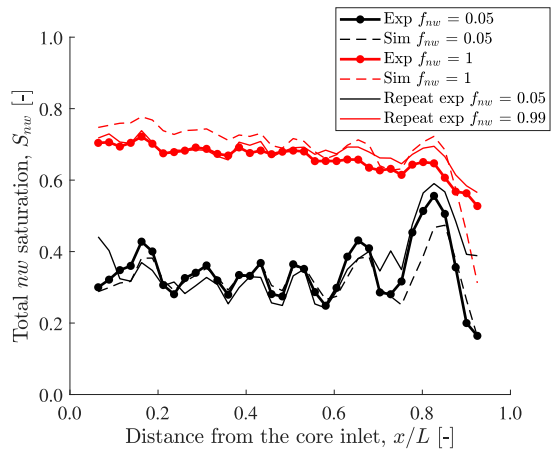

(c)

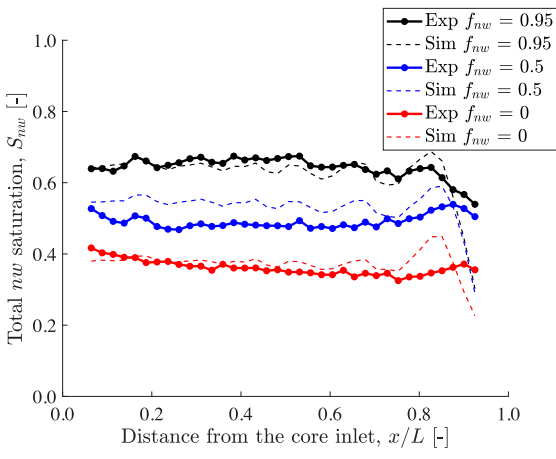

(e)

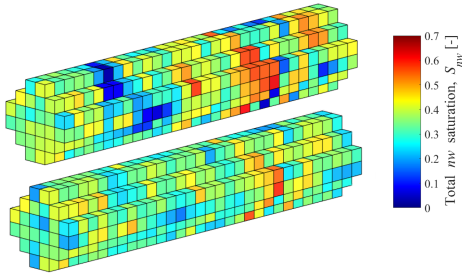

(b)

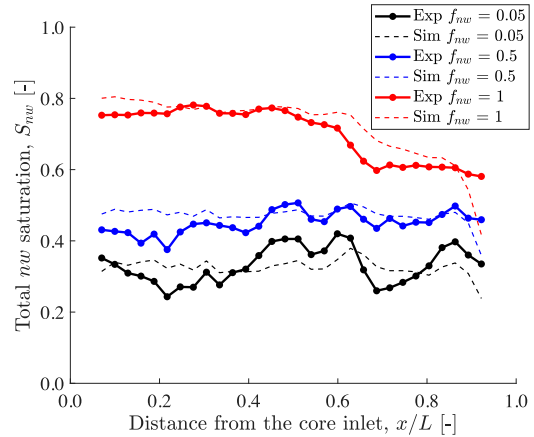

(d)

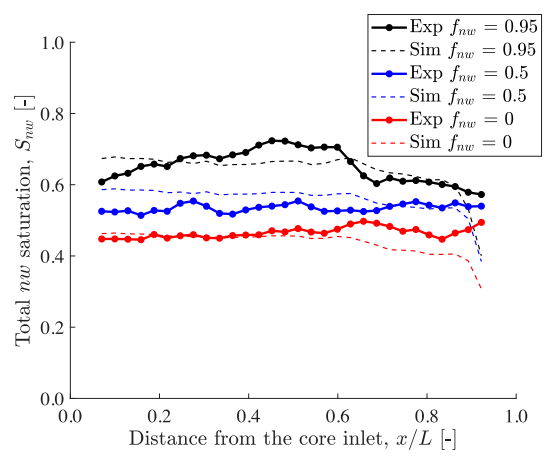

(f)

Figure 7. Simulated vs. experimental saturations. Left hand side - core 1, right hand side core 2. (a), (b) 3D non-wetting saturation rendering for drainage $f_{n w}=0.05$. Top - experiments, bottom - simulations. (c), (d) Slice average total non-wetting saturations during drainage. (e), (f) Slice average total non-wetting saturations during imbibition. 
impact primarily due to the capillary pressure hysteresis. During imbibition, the capillary pressure curves are much steeper than drainage, meaning spatial gradients are smaller and hence saturation heterogeneity is reduced. We note that the numerical model here is performing in a much more predictive manner than during drainage; the function parametrisation relies solely on the drainage data coupled with final trapping data, which is used with physical arguments to construct the imbibition functions. The capillary pressure heterogeneity during imbibition is scaled using the same parametrisation, equation (11), as that during drainage, and is able to reproduce the experimental saturation variation through the core.

Experimental and simulated relative permeabilities are shown in Figure 8. The relative permeability experimental error bars arise from the standard deviation in experimental pressure differential, which is most prominent at low wetting saturations due to the low pressure differential. Saturation error bars represent the standard deviation in slice average saturation from the whole core average, highlighting heterogeneity at low $f_{n w}$ and end effects at high $f_{n w}$. These uncertainties are generally larger than those arising from segmentation, see Figure 4. In Figure 8c, d we also display the repeat drainage experimental data in yellow, highlighting the consistency in relative permeability between measurements.

In Figure 8a, b we show the non-wetting relative permeability vs. total and connected saturation respectively, during drainage and imbibition. By considering the mobile, connected non-wetting saturation only, the observed hysteresis in the experimental relative permeability is completely removed. This directly validates conceptual models of connected pathway flow, whereby considering the same connected pathway saturation at low capillary number produces the same relative permeability (Berg et al., 2016; Picchi \& Battiato, 2018). The results here confirm this over multiple fractional flows, and in two distinct samples.

The intrinsic curves used for modelling both cores are identical, with $k_{r n w}\left(S_{w i r r}\right)$ $=0.8$. The non-wetting phase exponent $n=4.6$ here, which is close to the theoretical scaling for an invasion percolation displacement in 3D (Dietrich Stauffer, 1994; M. Blunt et al., 1992), i.e. $k_{r n w} \sim S_{n w}^{4.8}$. Indeed, the low fractional flows of non-wetting phase during drainage exhibit invasion percolation displacement, controlled by the pore-size distribution and the availability of pores and throats, see section 4.2. The wetting phase exponent is similar to the non-wetting phase at $m=4.4$; we note that during thin-film flow the exponent can be expected to scale as $k_{r, w} \sim S_{w}^{4}$ (Blunt, 2017), which is observed here at low wetting phase saturations.

The drainage core averaged relative permeabilities are well captured by the numerical model, with relative errors $<11 \%$. Core averaged saturations errors are also very low $<5 \%$. During imbibition the experimental data is generally less well predicted than drainage, with average saturation errors $\approx 10 \%$ and relative permeabilty errors $\approx 20 \%$. Despite the relative error in the simulated and experimental results, we note that they are largely in line with experimental uncertainties which are larger during the imbibition fractional flows. The numerical model is able to capture the key characteristics in the relative permeability deviation from the intrinsic curves, reflecting the effect of heterogeneity with relatively simple functional parametrisation based on the residual trapping. Note that drainage in the model was parametrised with an intrinsic drainage relative permeability curve, while imbibition is entirely parametrised by the fit trapping model.

The increase of the water relative permeability during imbibition is captured in the model using a decrease in the power law exponent, resulting in a good prediction of the experimental data. The slight raising in water relative permeability during imbibition has been seen in other experimental data (see C. Reynolds (2016); Shi et al. (2017); Akbarabadi and Piri (2012))), and could be an impact of the heterogeneity in the system. 


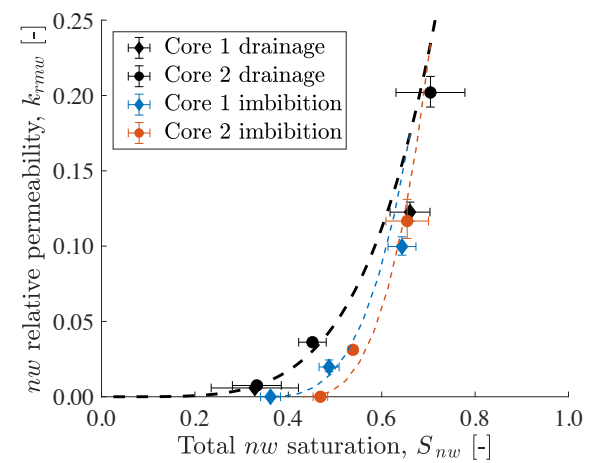

(a)

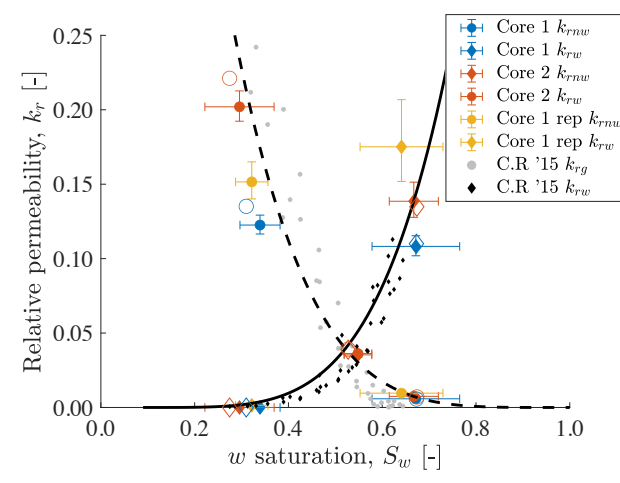

(c)

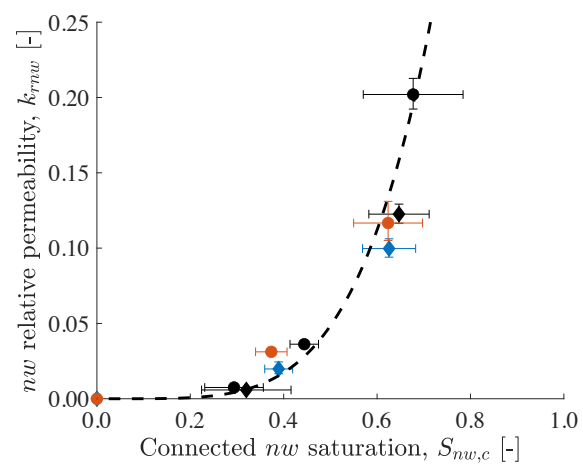

(b)

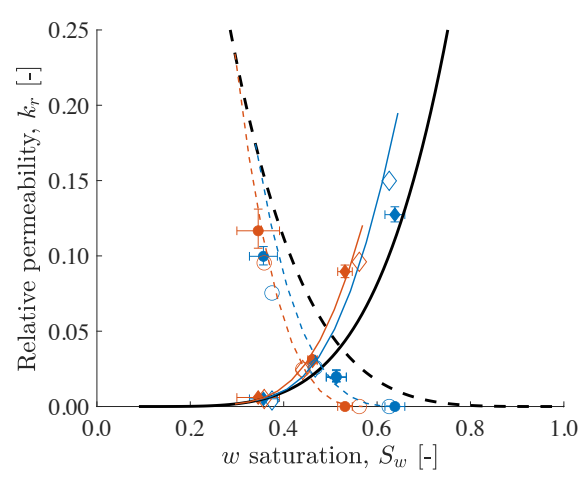

(d)

Figure 8. (a), (b) Experimental relative permeability during drainage and imbibition vs. total, and connected non-wetting saturations, respectively. (c), (d) Simulated and experimental drainage and imbibition relative permeabilities. Closed symbols are experimental data, open symbols are simulations. Solid and dashed lines are the intrinsic wetting and non-wetting relative permeabiltity functions, respectively. Black lines are for drainage, blue and orange lines are for imbibition with land $C_{l}=1.3$ and $C_{l}=0.9$ respectively. C. R. ' 15 data refers to (Reynolds \& Krevor, 2015). 


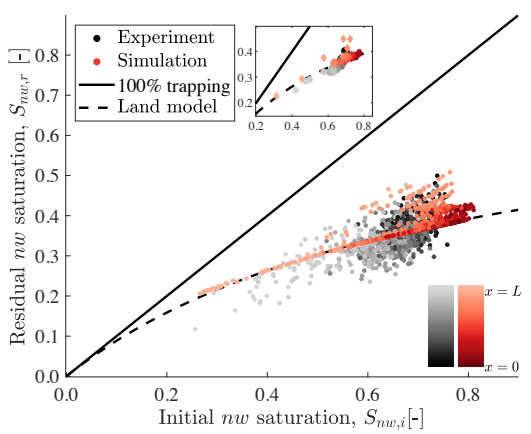

(a)

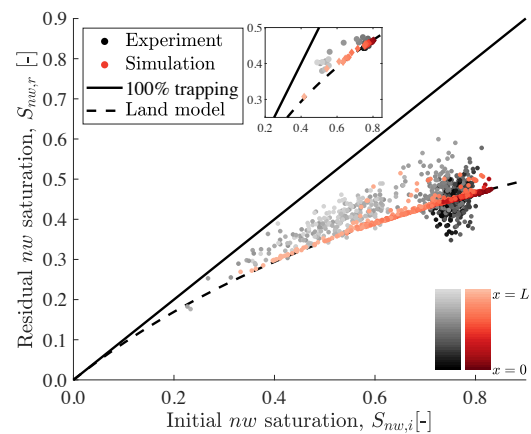

(b)

Figure 9. REV level initial-residual trapping results for the experiments and simulations. The zoom in box plots show the slice average trapping results. (a) Core 1 (b) Core 2.

Hysteresis appears more prominent in core 2, which could be a consequence of the enhanced snap-off trapping in the low porosity region, which causes the water to displace the non-wetting phase in cooperative pore filling events upstream. Filling larger pores here would result in enhanced conductivity through the system.

The deviations of both the experiments and simulated data points from the intrinsic curves reflect the impacts of rock heterogeneity. The proximity of the simulation points to the experimental points is a measure of the ability of the parameterised model to predict these impacts. The same intrinsic relative permeability is used for both cores, but the distinct spatial heterogeneities manifest in different equivalent relative permeabilities at the largest (core) averaging scale. The reduced impacts of heterogeneity during imbibiton have rendered the equivalent functions closer to the intrinsic, which are well predicted by the interpolated scanning functions using Killough's method (Killough, 1976.). This result has important implications for continuum scale modelling; for samples with similar global pore-size distributions the same intrinsic functions coupled with REV scale heterogeneities can be used to predict experimental observations, in the regime of connected pathway, low capillary number flow.

The magnitude of the capillary pressure heterogeneities are relatively low in the samples, with $\eta=\sigma\left(P_{e}\right) / \mu\left(P_{e}\right)=0.095$ and 0.080 for cores 1 and 2 respectively, similar to that reported for Berea cores $\eta \approx 0.1$ (Pini \& Benson, 2017). For stronger heterogeneities, such as that seen in reservoir cores (e.g. a Bunter core with $\eta \approx 0.17$ (Reynolds et al., 2018; Jackson et al., 2018)), or carbonate samples (e.g. Indiana limestone $\eta=$ 0.21 (Hejazi et al., 2019)) the relative permeability variation can be much lager, with orders of magnitude variation between different capillary number experiments (Reynolds et al., 2018).

The numerical model is also able to reproduce, to some extent, the observed trapping at $f_{n w}=0$, shown in Figure 9. Here, most of the simulated data points fall exactly on the trapping model used for the simulations, i.e. the trapping occurs primarily through snap-off. However, there are data points with non-wetting saturations higher than that given by the trapping model, indicating enhanced capillary heterogeneity trapping, as seen in the experiments.

Key to modelling this process during imbibition is having a finite, non-zero macroscopic capillary pressure at the residual non-wetting saturation (Jackson \& Krevor, 2019; Bech \& Frykman, 2018; Zahasky \& Benson, 2019), as shown in SI Figure 7. This finite capillary pressure is observed experimentally (Raeesi et al., 2014), but is often reduced 
to zero, or negative values by further reducing the wetting phase pressure relative to the non-wetting phase. There is generally no further production of non-wetting phase, and the end-point of the capillary pressure curve can be arbitrarily defined. For water-wet media, the end-point is often defined as $P_{s}=0$ (Pini \& Benson, 2017), but this leads to under-estimation of the amount of heterogeneity trapping in the system. If all points in the core can reduce to a capillary pressure of zero at the residual, essentially no gradients are observed in the capillary pressure, which inhibits any local heterogeneity trapping described above. The finite value of capillary pressure can also be negative for mixed or oil wet systems, and capillary pressure gradients can be sustained in the system (Nooruddin \& Blunt, 2017).

The simulation results here reveal that boundary conditions during imbibiton are important for the model to recreate the experimental observations, but are difficult to estimate from the experiments. In the experiments the end effects (i.e. tailing of the nonwetting phase towards the outlet) are reduced, or inverted during imbibition, and proper inclusion of these impacts in models is key to an accurate match. Here, we use the imbibition residual capillary pressure, $P_{s}$, as a constant capillary pressure for the boundary condition. Since the capillary pressure reduces significantly during imbibition, using a lower boundary condition is needed, otherwise artificial capillary pressure gradients are created in the core (i.e. if one uses the drainage entry pressure $P_{e}$ ). Using this condition, we are able to reduce the impact of end-effects compared to drainage, and correctly capture capillary pressure heterogeneity in the core, but we still underestimate the saturation at the outlet.

It is key to model hysteresis in capillary pressure to predict the variation in saturation profiles between drainage and imbibition and the impact of trapping (Jackson \& Krevor, 2019), but this is often neglected in core-scale modelling (Ni et al., 2019) and in larger field-scale modelling (Saadatpoor et al., 2010). In this work, through the combined use of pore-scale imaging and continuum scale modelling we have demonstrated a multi-scale approach to analyse immiscible displacement in porous media.

\section{Conclusions and future outlook}

In this work, we combine multi-scale X-ray observations of drainage and imbibition displacements with continuum numerical models to assess the REV for single and multiphase flow properties, the relationship between hysteresis and non-wetting phase fluid connectivity, and the role of small scale continuum property heterogeneities on the upscaled manifestation of relative permeability. The analysis was performed using a water wet fluid-rock system, decane and water, in a homogeneous and a heterogeneous Bentheimer sandstone rock core.

The cubic REV side length for both porosity and capillary pressure characteristic curves is $\approx 1.25 \mathrm{~mm}$ for both heterogeneous and homogeneous samples, with an uncertainty of $5 \%$. In contrast, the REV for saturation varies with fractional flow between $1.5 \mathrm{~mm}-5.4 \mathrm{~mm}$. It is key to utilise the knowledge of the REV in modelling, assessing uncertainty in model predictions based on observed fluctuations in the continuum property, and thus removing the arbitrariness of the REV definition. In the low capillary number regime analysed in this work, capillary pressure heterogeneity at the REV scale controlled the fluid distribution, and must be accounted for in the interpretation of corescale observations.

We utilise the Land trapping model to predict fluid connectivity and hysteresis during multiple states of imbibition and compared these with pore scale observations of fluid connectivity. We find that in homogeneous media, the Land trapping model can successfully predict fluid connectivity at a range of imbibition states, parametrised purely on the initial-residual data. In heterogeneous media, REV scale heterogeneities also have 
to be incorporated to capture the larger scale capillary heterogeneity trapping. We also find that through incorporation of the connected saturation, hysteresis in the experimental non-wetting relative permeability is entirely removed. Fluid morphology is increasingly viewed as essential for a rigorous representation of upscaled flow properties; here we validate that the connectivity is sufficient to fully parametrise the continuum flow properties across displacement pathways during connected pathway flow in homogeneous media. Modest continuum scale heterogeneity results in an added requirement of multi scale modelling in which the continuum scale heterogeneity is represented, in addition to the parametrisation of pore scale fluid connectivity.

We develop 3D continuum scale numerical models of the rock cores which are largely able to predict the impacts of small scale heterogeneities on the upscaled saturations and relative permeability. The models for both samples utilised the same intrinsic functions with different heterogeneity structures, and were able to capture the relative variation in upscaled relative permeabilties and fluid distributions. The models confirm that both heterogeneity and hysteresis in the capillary pressure characteristics are key to the upscaled manifestation of relative permeability, even at the cm-scale of the rock cores used in this work. This suggests that for physics-based modelling of subsurface multiphase flow, a workflow is required which successfully accounts for the upscaled impacts of these properties.

The experimental dataset presented in this paper, and available for download online, provides an excellent benchmark for developing and validating continuum scale modelling approaches. Key to future developments of continuum models are the thorough inclusion of connectivity evolution during both drainage and imbibition (i.e. the roof snapoff mechanism that can occur during primary drainage), and the incorporation of these effects with REV scale heterogeneities. Further developments are also needed to incorporate multi-scale REV heterogeneities, such as those occurring in carbonate rocks (Hejazi et al., 2019).

Alongside this, continuum models must also incorporate the impacts of high $N_{c p}$ flows, that lead to intermittent fluid connectivity (Spurin et al., 2019a, 2019b) and enhanced ganglion dynamics (Rücker et al., 2015). While the use of extended Darcy's law with REV scale heterogeneities is largely sufficient for capturing the flow regimes presented in this paper, and is sufficient for many field-scale applications, it is likely that extended models, possibly based on the fundamental thermodynamics of the system may be needed to capture the full manifestation of immiscible pore-scale physics at the continuum scale (Gray \& Miller, 2005; Niessner et al., 2011). It is our hope that this work and dataset will serve as a base to advance such endeavours, leading to predictive, multiscale continuum models.

\section{Acknowledgements}

We gratefully acknowledge funding from the Shell Digital Rocks programme at Imperial College London and the Natural Environment Research Council (Grant number: NE/N016173/1). We acknowledge Computer Modelling Group (CMG) for providing access to IMEX. We thank Ab. Coorn from Shell for sample preparation and Steffen Berg for helpful comments. We also thank Martin Blunt, Branko Bijeljic and Ronny Pini for insightful discussion. We thank Chris Zahasky for useful comments on the draft paper. The authors have no conflicts of interests.

Data associated with this work is available online in two locations, suitable for different purposes. The full dataset, with all associated files (raw X-Ray CT imagery and segmented data, post-processed porosity and saturation Matlab data, CMG simulation files and Matlab processing files) is hosted on the BGS National Geoscience Data Centre, ID \#130625 at dx.doi.org/10.5285/5f899de8-4085-4370-a45e-e613f27e8f1d. Due to 
the large data size $(\approx 2.5 \mathrm{~Tb}$ in total for all X-Ray $\mathrm{CT}$ data; each full core $6 \mu \mathrm{m}$ image is $\approx 80 \mathrm{~Gb}$ ), a sub-volume image data set is also available on the Digital Rocks Portal, project 229, at dx.doi.org/10.17612/KT0B-SZ28. Here we provide 50 subvolumes of each image, with size $\approx 1.5 \mathrm{~Gb}$, making data manipulation and handling less computationally demanding. These subvolumes can be concatenated to produce the full image.

\section{References}

Akbarabadi, M., \& Piri, M. (2012). Relative permeability hysteresis and capillary trapping characteristics of supercritical $\mathrm{CO}_{2} /$ brine systems: an experimental study at reservoir conditions. Advances in Water Resources, 52, 190-206. doi: 10.1016/j.advwatres.2012.06.014

Al-Raoush, R., \& Papadopoulos, A. (2010). Representative elementary volume analysis of porous media using x-ray computed tomography. Powder Technology, 200(1-2), 69-77. doi: 10.1016/j.powtec.2010.02.011

AlRatrout, A., Blunt, M. J., \& Bijeljic, B. (2018). Spatial correlation of contact angle and curvature in pore-space images. Water Resources Research, 54 (9), 6133-6152. doi: 10.1029/2017wr022124

Andrew, M., Bijeljic, B., \& Blunt, M. J. (2014a). Pore-by-pore capillary pressure measurements using x-ray microtomography at reservoir conditions: Curvature, snap-off, and remobilization of residual CO2. Water Resources Research, 50(11), 8760-8774. doi: 10.1002/2014wr015970

Andrew, M., Bijeljic, B., \& Blunt, M. J. (2014b). Pore-scale contact angle measurements at reservoir conditions using x-ray microtomography. Advances in Water Resources, 68, 24-31. doi: 10.1016/j.advwatres.2014.02.014

Andrew, M., Menke, H., Blunt, M. J., \& Bijeljic, B. (2015). The imaging of dynamic multiphase fluid flow using synchrotron-based x-ray microtomography at reservoir conditions. $\quad$ Transport in Porous Media, 110(1). doi: 10.1007/s11242-015-0553-2

Armstrong, R., Georgiadis, A., Ott, H., Klemin, D., \& Berg, S. (2014). Critical capillary number: Desaturation studied with fast X-ray computed microtomography. Geophysical Research Letters, 41, 55-60. doi: 10.1002/2013gl058075

Armstrong, R. T., McClure, J. E., Robins, V., Liu, Z., Arns, C. H., Schlüter, S., \& Berg, S. (2018). Porous media characterization using minkowski functionals: Theories, applications and future directions. Transport in Porous Media. doi: 10.1007/s11242-018-1201-4

Arns, C., Knackstedt, M., \& Martys, N. (2005). Cross-property correlations and permeability estimation in sandstone. Physical Review E, 72(4). doi: 10.1103/ physreve.72.046304

Aveyard, R., \& Saleem, S. M. (1976). Interfacial tensions at alkane-aqueous electrolyte interfaces. Journal of the Chemical Society, Faraday Transactions 1: Physical Chemistry in Condensed Phases, 72(0), $1609 . \quad$ doi: 10.1039/f19767201609

Bear, J. (1989). Dynamics of fluids in porous media. Dover Publications Inc.

Bech, N., \& Frykman, P. (2018). Trapping of buoyancy-driven CO2 during imbibition. International Journal of Greenhouse Gas Control, 78, 48-61. doi: 10 .1016/j.ijggc.2018.06.018

Berg, S., Ott, H., Klapp, S. A., Schwing, A., Neiteler, R., Brussee, N., .. Stampanoni, M. (2013). Real-time 3d imaging of haines jumps in porous media flow. Proceedings of the National Academy of Sciences, 110(3755). doi: 10.1073 pnas. 1221373110

Berg, S., Rucker, M., Ott, H., Georgiadis, A., H. van der Linde, F. E., Kersten, M., ... Wiegmann, A. (2016). Connected pathway relative permeability from pore-scale imaging of imbibition. Advances in Water Resources, 90(24). doi: 10.1016/j.advwatres.2016.01.010 
Blunt, M., King, M. J., \& Scher, H. (1992). Simulation and theory of two-phase flow in porous media. Physical Review A, 46(12), 7680-7699. doi: 10.1103/physreva .46 .7680

Blunt, M. J. (2017). Multiphase flow in permeable media: A pore-scale perspective. Cambridge University Press.

Blunt, M. J., Bijeljic, B., Dong, H., Gharbi, O., Iglauer, S., Mostaghimi, P., ... Pentland, C. (2013). Pore-scale imaging and modelling. Advances in Water Resources, 51(197). doi: 10.1016/j.advwatres.2012.03.003

Bourgeat, A. (1997). Two-phase flow. In Homogenization and porous media. interdisciplinary applied mathematics, vol 6. (pp. 95-127). Springer New York. doi: 10.1007/978-1-4612-1920-0_5

Brooks, R. H., \& Corey, A. T. (1964). Hydraulic properties of porous media. In $H y$ drology paper no. 3.

Buades, A., Coll, B., \& Morel, J.-M. (2007). Nonlocal image and movie denoising. International Journal of Computer Vision, 76 (2), 123-139. doi: 10.1007/ s11263-007-0052-1

Carlson, F. M. (1981). Simulation of relative permeability hysteresis to the nonwetting phase. In SPE annual technical conference and exhibition. Society of Petroleum Engineers. doi: 10.2118/10157-ms

Chaouche, M., Rakotomalala, N., Salin, D., Xu, B., \& Yortsos, Y. (1994). Capillary effects in drainage in heterogeneous porous media: continuum modelling, experiments and pore network simulations. Chemical Engineering Science, 49(15), 2447-2466. doi: 10.1016/0009-2509(94)e0040-w

Chaouche, M., Rakotomalala, N., Salin, D., \& Yortsos, Y. C. (1993). Capillary effects in immiscible flows in heterogeneous porous media. Europhysics Letters (EPL), 21(1), 19-24. doi: 10.1209/0295-5075/21/1/004

Corbett, P. W. M., Ringrose, P. S., Jensen, J. L., \& Sorbie, K. S. (1992). Laminated clastic reservoirs: The interplay of capillary pressure and sedimentary architecture. In 6rth Annual Technical Conference and Exhibition of the Society of Petroleum Engineers, Washington D.C., October 4-7, 1992. $\quad$ doi: 10.2118/24699-ms

Costanza-Robinson, M. S., Estabrook, B. D., \& Fouhey, D. F. (2011). Representative elementary volume estimation for porosity, moisture saturation, and air-water interfacial areas in unsaturated porous media: Data quality implications. Water Resources Research, 47(7). doi: 10.1029/2010wr009655

Dietrich Stauffer, A. A. (1994). Introduction to percolation theory. Taylor \& Francis Ltd.

Fernandes, J., Appoloni, C., \& Fernandes, C. (2012). Determination of the representative elementary volume for the study of sandstones and siltstones by X-Ray microtomography. Materials Research, 15(4), 662-670. doi: 10.1590/s1516-14392012005000081

Finsterle, S., Sonnenthal, E. L., \& Spycher, N. (2014, apr). Advances in subsurface modeling using the TOUGH suite of simulators. Computers $\&$ Geosciences, 65, 2-12. doi: 10.1016/j.cageo.2013.06.009

Gao, Y., Lin, Q., Bijeljic, B., \& Blunt, M. J. (2017). X-ray microtomography of intermittency in multiphase flow at steady state using a differential imaging method. Water Resources Research, 53(12), 10274-10292. doi: 10.1002/2017wr021736

Georgiadis, A., Maitland, G., Trusler, J. P. M., \& Bismarck, A. (2011). Interfacial tension measurements of the (h2o - - n-decane - - $\left.\mathrm{CO}_{2}\right)$ ternary system at elevated pressures and temperatures. Journal of Chemical \& Engineering Data, 56(12), 4900-4908. doi: 10.1021/je200825j

Ghafri, S. A., Maitland, G. C., \& Trusler, J. P. M. (2012). Densities of aqueous $\mathrm{MgCl} 2(\mathrm{aq}), \mathrm{CaCl} 2(\mathrm{aq}), \mathrm{KI}(\mathrm{aq}), \mathrm{NaCl}(\mathrm{aq}), \mathrm{KCl}(\mathrm{aq}), \mathrm{AlCl} 3(\mathrm{aq})$, and (0.964 $\mathrm{NaCl}+0.136 \mathrm{KCl})(\mathrm{aq})$ at temperatures between $(283$ and 472$) \mathrm{k}$, pressures up 
to $68.5 \mathrm{MPa}$, and molalities up to $6 \mathrm{~mol} / \mathrm{kg}$. Journal of Chemical ES Engineering Data, 57(4), 1288-1304. doi: 10.1021/je2013704

Goldsack, D. E., \& Franchetto, R. (1977). The viscosity of concentrated electrolyte solutions. i. concentration dependence at fixed temperature. Canadian Journal of Chemistry, 55(6), 1062-1072. doi: 10.1139/v77-148

Gray, W. G., \& Miller, C. T. (2005). Thermodynamically constrained averaging theory approach for modeling flow and transport phenomena in porous medium systems: 1. motivation and overview. Advances in Water Resources, 28(2), 161-180. doi: 10.1016/j.advwatres.2004.09.005

Haines, W. B. (1930). Studies in the physical properties of soil. v. the hysteresis effect in capillary properties, and the modes of moisture distribution associated therewith. The Journal of Agricultural Science, 20(1), 97-116. doi: $10.1017 / \mathrm{s} 002185960008864 \mathrm{x}$

Halisch, M. (2013). The rev challenge - estimating representative elementary volumes and porous rock inhomogeneity from high resolution micro-CT data sets. In International symposium of the society of core analysts sca2013-069.

Hassanizadeh, M., \& Gray, W. G. (1980). General conservation equations for multiphase systems: 3. constitutive theory for porous media flow. Advances in Water Resources, 3(1), 25-40. doi: 10.1016/0309-1708(80)90016-0

Hejazi, S. A. H., Shah, S., \& Pini, R. (2019, jun). Dynamic measurements of drainage capillary pressure curves in carbonate rocks. Chemical Engineering Science, 200, 268-284. doi: 10.1016/j.ces.2019.02.002

Herring, A. L., Gilby, F. J., Li, Z., McClure, J. E., Turner, M., Veldkamp, J. P., ... Sheppard, A. P. (2018). Observations of nonwetting phase snapoff during drainage. Advances in Water Resources, 121, 32-43. doi: 10.1016/j.advwatres.2018.07.016

Herring, A. L., Harper, E. J., Andersson, L., Sheppard, A., Bay, B. K., \& Wildenschild, D. (2013). Effect of fluid topology on residual nonwetting phase trapping: Implications for geologic CO2 sequestration. Advances in Water Resources, 62, 47-58. doi: 10.1016/j.advwatres.2013.09.015

Hilfer, R., \& Lemmer, A. (2015). Differential porosimetry and permeametry for random porous media. Physical Review E, 92(1). doi: 10.1103/physreve.92 .013305

Honarpour, M., Cullick, A., Saad, N., \& Humphreys, N. $\quad$ (1995). $\quad$ Effect of rock heterogeneity on relative permeability: Implications for scaleup. Journal of Petroleum Technology, 47(11), 980-986. doi: 10.2118/29311-pa

Jackson, S. J., Agada, S., Reynolds, C. A., \& Krevor, S. (2018). Characterizing drainage multiphase flow in heterogeneous sandstones. Water Resources Research, 54 (4), 3139-3161. doi: 10.1029/2017wr022282

Jackson, S. J., \& Krevor, S. (2019). Characterization of hysteretic multiphase flow from the $\mathrm{mm}$ to $\mathrm{m}$ scale in heterogeneous rocks. E3S Web of Conferences, 89, 02001. doi: $10.1051 / \mathrm{e} 3$ sconf $/ 20198902001$

Jensen, J. L., Corbett, P. W. M., Pickup, G. E., \& Ringrose, P. S. (1996). Permeability semivariograms, geological structure, and flow performance. Mathematical Geology, 28(4), 419-435. doi: 10.1007/bf02083654

Jerauld, G. R. (1997). Prudhoe bay gas/oil relative permeability. SPE Reservoir Engineering, 12(01), 66-73. doi: 10.2118/35718-pa

Joekar-Niasar, V., Hassanizadeh, S. M., \& Leijnse, A. (2007). Insights into the relationships among capillary pressure, saturation, interfacial area and relative permeability using pore-network modeling. Transport in Porous Media, 74 (2), 201-219. doi: 10.1007/s11242-007-9191-7

Jones, A., C, A., Sheppard, A., Hutmacher, D., Milthorpe, B., \& Knackstedt, M.

(2007). Assessment of bone ingrowth into porous biomaterials using MICROCT. Biomaterials, 28(15), 2491-2504. doi: 10.1016/j.biomaterials.2007.01.046

Killough, J. E. (1976.). Reservoir simulation with history-dependent saturation func- 
tions. Society of Petroleum Engineers Journal, 37-48.

Kogure, T., Zhang, Y., Nishizawa, O., \& Xue, Z. (2017, aug). Migration mode of brine and supercritical CO2 during steady-state relative permeability measurements at very slow fluid flow velocity. Geophysical Journal International. doi: $10.1093 /$ gji/ggx335

Krause, M., \& Benson, S. (2015). Accurate determination of characteristic relative permeability curves. Advances in Water Resources, 83, 376-388. doi: 10.1016/ j.advwatres.2015.07.009

Krause, M., Krevor, S., \& Benson, S. (2013). A procedure for the accurate determination of sub-core scale permeability distributions with error quantification. Transport in Porous Media, 93(3), 565-588. doi: 10.1007/s11242-013-0161-y

Krevor, S., Blunt, M. J., Benson, S. M., Pentland, C. H., Reynolds, C., Al-Menhali, A., \& Niu, B. (2015, sep). Capillary trapping for geologic carbon dioxide storage - from pore scale physics to field scale implications. International Journal of Greenhouse Gas Control, 40, 221-237. doi: 10.1016/j.ijggc.2015.04.006

Krevor, S., Blunt, M. J., Trusler, J. P. M., \& Simone, S. D. S. $\quad$ (2019). An introduction to subsurface $\mathrm{CO}_{2}$ storage. In N. Mac Dowell \& M. Bui (Eds.), Carbon capture and storage.

Krevor, S. C. M., Pini, R., Li, B., \& Benson, S. M. (2011). Capillary heterogeneity trapping of $\mathrm{CO}_{2}$ in a sandstone rock at reservoir conditions. Geophysical Research Letters, 38, L15401. doi: 10.1029/2011gl048239

Land, C. (1968). Calculation of imbibition relative permeability for two- and threephase flow from rock properties. Society of Petroleum Engineers Journal, 149156. doi: 10.2118/1942-pa

Lenormand, R., Zarcone, C., \& Sarr, A. (1983). Mechanisms of the displacement of one fluid by another in a network of capillary ducts. Journal of Fluid Mechanics, $135(-1)$, 337. doi: 10.1017/s0022112083003110

Lin, Q., Al-Khulaifi, Y., Blunt, M. J., \& Bijeljic, B. （2016). Quantification of sub-resolution porosity in carbonate rocks by applying high-salinity contrast brine using x-ray microtomography differential imaging. Advances in Water Resources, 96, 306-322. doi: 10.1016/j.advwatres.2016.08.002

Lin, Q., Bijeljic, B., Berg, S., Pini, R., Blunt, M. J., \& Krevor, S. (2019, jun). Minimal surfaces in porous media: Pore-scale imaging of multiphase flow in an altered-wettability bentheimer sandstone. Physical Review E, 99(6). doi: 10.1103/physreve.99.063105

Lin, Q., Bijeljic, B., Pini, R., Blunt, M. J., \& Krevor, S. (2018). Imaging and measurement of pore-scale interfacial curvature to determine capillary pressure simultaneously with relative permeability. Water Resources Research, 54 (9), 7046-7060. doi: 10.1029/2018wr023214

Lin, Q., Bijeljic, B., Rieke, H., \& Blunt, M. J. (2017). Visualization and quantification of capillary drainage in the pore space of laminated sandstone by a porous plate method using differential imaging x-ray microtomography. Water Resources Research, 53(8), 7457-7468. doi: 10.1002/2017wr021083

Linstrom, P. (1997). Nist chemistry webbook, nist standard reference database 69. National Institute of Standards and Technology. doi: 10.18434/t4d303

McClure, J. E., Armstrong, R. T., Berrill, M. A., Schlüter, S., Berg, S., Gray, W. G., \& Miller, C. T. (2018). Geometric state function for two-fluid flow in porous media. Physical Review Fluids, 3(8). doi: 10.1103/physrevfluids.3.084306

McClure, J. E., Berrill, M. A., Gray, W. G., \& Miller, C. T. (2016). Influence of phase connectivity on the relationship among capillary pressure, fluid saturation, and interfacial area in two-fluid-phase porous medium systems. Physical Review E, 94(3). doi: 10.1103/physreve.94.033102

McPhee, C., Reed, J., \& Zubizarreta, I. (2015). Core analysis: A best practice guide. In J. Cubitt (Ed.), Developments in petroleum science (Vol. 64).

Morrow, N. R. (1970). Physics and thermodynamics of capillary action in 
porous media. Industrial \& Engineering Chemistry, 62(6), 32-56. doi: $10.1021 /$ ie50726a006

Muskat, M., \& Meres, M. W. (1936). The flow of heterogeneous fluids through porous media. Physics, 7(9), 346-363. doi: 10.1063/1.1745403

Ni, H., Boon, M., Garing, C., \& Benson, S. M. (2019). Predicting CO2 residual trapping ability based on experimental petrophysical properties for different sandstone types. International Journal of Greenhouse Gas Control, 86, 158176. doi: $10.1016 /$ j.ijggc.2019.04.024

Niessner, J., Berg, S., \& Hassanizadeh, S. M. (2011). Comparison of two-phase darcy's law with a thermodynamically consistent approach. Transport in Porous Media, 88(1), 133-148. doi: 10.1007/s11242-011-9730-0

Nooruddin, H. A., \& Blunt, M. J. $\quad$ (2017, nov). Large-scale invasion percolation with trapping for upscaling capillary-controlled darcy-scale flow. Transport in Porous Media, 121(2), 479-506. doi: 10.1007/s11242-017-0960-7

Nordahl, K., \& Ringrose, P. S. (2008). Identifying the representative elementary volume for permeability in heterolithic deposits using numerical rock models. Mathematical Geosciences, 40(7), 753-771. doi: 10.1007/s11004-008-9182-4

Oak, M. (1980). Three-phase relative permeability of water-wet berea. In Spe/doe seventh symposium on enhanced oil recovery, tulsa, oklahoma. doi: $10.2118 / 20183-\mathrm{ms}$

Peksa, A., Wolf, K.-A., \& Zitha, P. (2015). Bentheimer sandstone revisited for experimental purposes. Marine and Petroleum Geology, 67, 701-719. doi: 10 .1016/j.marpetgeo.2015.06.001

Picchi, D., \& Battiato, I. (2018). The impact of pore-scale flow regimes on upscaling of immiscible two-phase flow in porous media. Water Resources Research, 54(9), 6683-6707. doi: 10.1029/2018wr023172

Picchi, D., \& Battiato, I. (2019). Relative permeability scaling from pore-scale flow regimes. Water Resources Research, 55(4), 3215-3233. doi: 10.1029/ 2018wr024251

Pini, R., \& Benson, S. (2013). Characterization and scaling of mesoscale heterogeneities in sandstones. Geophysical Research Letters, 40, 3903-3908. doi: 10 $.1002 / \mathrm{grl} .50756$

Pini, R., \& Benson, S. M. (2017). Capillary pressure heterogeneity and hysteresis for the supercritical $\mathrm{CO}_{2}$ /water system in a sandstone. Advances in Water Resources, 108, 277-292. doi: 10.1016/j.advwatres.2017.08.011

Pini, R., Krevor, S., \& Benson, S. (2012). Capillary pressure and heterogeneity for the $\mathrm{CO}_{2}$ /water system in sandstone rocks at reservoir conditions. Advances in Water Resources, 38, 48-59. doi: 10.1016/j.advwatres.2011.12.007

Porter, M. L., Wildenschild, D., Grant, G., \& Gerhard, J. I. (2010). Measurement and prediction of the relationship between capillary pressure, saturation, and interfacial area in a NAPL-water-glass bead system. Water Resources Research, 46(8). doi: 10.1029/2009wr007786

Pyrak-Nolte, L. J., Nolte, D. D., Chen, D., \& Giordano, N. J. (2008). Relating capillary pressure to interfacial areas. Water Resources Research, 44(6). doi: 10 $.1029 / 2007 w r 006434$

Raeesi, B., Morrow, N. R., \& Mason, G. (2014). Capillary pressure hysteresis behavior of three sandstones measured with a multistep outflow-inflow apparatus. Vadose Zone Journal, 13(3), 0. doi: 10.2136/vzj2013.06.0097

Razavi, M. R., Muhunthan, B., \& Hattamleh, O. A. (2007). Representative elementary volume analysis of sands using x-ray computed tomography. Geotechnical Testing Journal, 30(3), 100164. doi: 10.1520/gtj100164

Reeves, P. C., \& Celia, M. A. (1996, aug). A functional relationship between capillary pressure, saturation, and interfacial area as revealed by a porescale network model. Water Resources Research, 32(8), 2345-2358. doi: 10.1029/96wr01105 
Reynolds, C. (2016). Two-phase flow behaviour and relative permeability between co2 and brine in sandstones at the pore and core scales (Ph.D. thesis). Imperial College London.

Reynolds, C. A., Blunt, M. J., \& Krevor, S. (2018). Multiphase flow characteristics of heterogeneous rocks from CO2 storage reservoirs in the united kingdom. Water Resources Research, 54(2), 729-745. doi: 10.1002/2017wr021651

Reynolds, C. A., \& Krevor, S. C. (2015). Characterizing flow behaviour for gas injection: Relative permeability of $\mathrm{CO}_{2}$-brine and $\mathrm{N}_{2}$-water in heterogeneous rocks. Water Resources Research, 51(12), 9464-9489. doi: 10.1002/2015wr018046

Reynolds, C. A., Menke, H., Andrew, M., Blunt, M. J., \& Krevor, S. (2017). Dynamic fluid connectivity during steady-state multiphase flow in a sandstone. Proceedings of the National Academy of Sciences, 114(31), 8187-8192. doi: $10.1073 /$ pnas. 1702834114

Ringrose, P., \& Bentley, M. (2015). Reservoir model design. a practitioners guide. Springer, Dordrecht.

Ringrose, P., \& Corbett, P. (1994). Controls on two-phase fluid flow in heterogeneous sandstones. Geological Society, London, Special Publications, 78, 141150. doi: 10.1144/gsl.sp.1994.078.01.12

Ringrose, P., Sorbie, K., Corbett, P., \& Jensen, J. (1993). Immiscible flow behaviour in laminated and cross-bedded sandstones. Journal of Petroleum Science and Engineering, 9, 103-124. doi: 10.1016/0920-4105(93)90071-1

Ruppert, L. F., Sakurovs, R., Blach, T. P., He, L., Melnichenko, Y. B., Mildner, D. F. R., \& Alcantar-Lopez, L. (2013). A USANS/SANS study of the accessibility of pores in the barnett shale to methane and water. Energy 83 Fuels, 27(2), 772-779. doi: 10.1021/ef301859s

Rücker, M., Berg, S., Armstrong, R. T., Georgiadis, A., Ott, H., Schwing, A., ... Kersten, M. (2015). From connected pathway flow to ganglion dynamics. Geophysical Research Letters, 42(10), 3888-3894. doi: 10.1002/2015gl064007

Saadatpoor, E., Bryant, S. L., \& Sepehrnoori, K. (2010). New trapping mechanism in carbon sequestration. Transport in Porous Media, 82, 3-17.

Schwartz, M. O. (2009, jan). Modelling groundwater contamination above the asse 2 medium-level nuclear waste repository, germany. Environmental Earth Sciences, 59(2), 277-286. doi: 10.1007/s12665-009-0025-5

Shi, S., Belhaj, H., \& Bera, A. (2017). Capillary pressure and relative permeability correlations for transition zones of carbonate reservoirs. Journal of Petroleum Exploration and Production Technology, 8(3), 767-784. doi: 10.1007/s13202-017-0384-5

Shook, M., Li, D., \& Lake, L. (1992). Scaling immiscible flow through permeable media by inspectional analysis. In Situ, 16(4), 311-349. doi: 10.1016/0148 -9062(93)91860-1

Singh, K., Bijeljic, B., \& Blunt, M. J. (2016). Imaging of oil layers, curvature and contact angle in a mixed-wet and a water-wet carbonate rock. Water Resources Research, 52(3), 1716-1728. doi: 10.1002/2015wr018072

Spiteri, E. J., Juanes, R., Blunt, M. J., \& Orr, F. M. (2008). A new model of trapping and relative permeability hysteresis for all wettability characteristics. SPE Journal, 13(03), 277-288. doi: 10.2118/96448-pa

Spurin, C., Bultreys, T., Bijeljic, B., Blunt, M. J., \& Krevor, S. (2019a). Intermittent fluid connectivity during two-phase flow in a heterogeneous carbonate rock. Phys. Rev. E, 100, 043103. doi: 10.1103/PhysRevE.100.043103

Spurin, C., Bultreys, T., Bijeljic, B., Blunt, M. J., \& Krevor, S. $\quad$ (2019b). Mechanisms controlling fluid breakup and reconnection during two-phase flow in porous media. Phys. Rev. E, 100, 043115. doi: 10.1103/PhysRevE.100 .043115

Todd, D. K. (2005). Groundwater hydrology. John Wiley \& Sons.

Trevisan, L., Pini, R., nd J.T. Birkholzer, A. C., Zhou, Q., González-Nicolás, A., \& 
Illangasekare, T. (2017). Imaging and quantification of spreading and trapping of carbon dioxide in saline aquifers using meter-scale laboratory experiments. Water Resources Research, 53(1), 485-502. doi: 10.1002/2016wr019749

Tuller, M., \& Or, D. (2001). Hydraulic conductivity of variably saturated porous media: Film and corner flow in angular pore space. Water Resources Research, 37(5), 1257-1276. doi: 10.1029/2000wr900328

Virnovsky, G., Friis, H., \& Lohne, A. (2004). A steady-state upscaling approach for immiscible two-phase flow. Transport in Porous Media, 54, 167-192.

Walsh, M., \& Lake, L. W. (2003). A generalized approach to primary hydrocarbon recovery of petroleum exploration \& production. Elsevier Science.

Whitaker, S. (1986). Flow in porous media II: The governing equations for immiscible, two-phase flow. Transport in Porous Media, 1(2), 105-125. doi: 10.1007/ bf00714688

Yoon, H., \& Dewers, T. A. (2013, aug). Nanopore structures, statistically representative elementary volumes, and transport properties of chalk. Geophysical Research Letters, 40(16), 4294-4298. doi: 10.1002/grl.50803

Zahasky, C., \& Benson, S. M. (2019, nov). Spatial and temporal quantification of spontaneous imbibition. Geophysical Research Letters, 46(21), 11972-11982. doi: $10.1029 / 2019 \mathrm{gl0} 104532$

Zhang, D., Zhang, R., Chen, S., \& Soll, W. (2000). Pore scale study of flow in porous media: Scale dependency, REV, and statistical REV. Geophysical Research Letters, 27(8), 1195-1198. doi: 10.1029/1999gl011101

Zhou, D., Fayers, F., \& Orr Jr, F. $\quad$ (1981). Scaling of multiphase flow in simple heterogeneous porous media. In 56th annual fall technical conference and exhibition of the society of petroleum engineers of aime, san antonio, texas, october 5-7, 1981. doi: 10.2118/27833-pa 